\title{
Quantization of the Bianchi type-IX model in N=1 Supergravity in the Presence of Supermatter
}

\author{
P.V. Moniz* \\ Department of Applied Mathematics and Theoretical Physics \\ University of Cambridge \\ Silver Street, Cambridge \\ CB3 9EW, UK
}

\begin{abstract}
The general theory of $\mathrm{N}=1$ supergravity with supermatter is applied to a Bianchi type IX diagonal model. The supermatter is constituted by a complex scalar field and its spin- $\frac{1}{2}$ fermionic partners. The Kähler geometry is chosen to be a two-dimensional flat one. The Lorentz invariant Ansatz for the wave function of the universe is taken to be as simple as possible in order to obtain new solutions. The set of differential equations derived from the quantum constraints are analysed in two different cases: if the supermatter terms include an analytical potential or not. In the latter the wave function is found to have a simple form.
\end{abstract}

PACS numbers: 04.60.+ $n, 04.65 .+e, 98.80 . \mathrm{Hw}$

\footnotetext{
* e-mail address: prlvm10@amtp.cam.ac.uk
} 


\section{Introduction}

The subjects of supersymmetric quantum gravity and cosmology have achieved a number of interesting results and conclusions during the last ten years or so. Several approaches may be found in the literature, namely the triad ADM canonical formulation $[1-26,62,63]$, the $\sigma$-model supersymmetric extension in quantum cosmology [27-31] and another approach based on Ashtekar variables [32-40]. Quite recently, important contributions have also been made $[22,23,24]$, which point towards a well desired revival of the field. A review on these subjects is currently in preparation [41].

The canonical quantization framework of $\mathrm{N}=1$ (pure) supergravity was presented in ref. [1], following $[42,43]$. It was pointed out that it would be sufficient, in finding a physical state, to solve the Lorentz and supersymmetry constraints of the theory because the algebra of constraints of the theory leads to anti-commutation relations implying that a physical wave functional $\Psi$ will also obey the Hamiltonian constraints $[1,42]$. The factor ordering of the Hamiltonian constraints is determined by the anti-commutation relations of the supersymmetry constraints. Namely, it will depend on how we order the fermionic derivatives in the supersymmetry constraints, which are enforced by the ordering in the spinorial form of the gravitational momentum. More precisely, such factor ordering implies that supersymmetry constraints should describe the left and right handed supersymmetry transformations (cf. ref.[1]) (when considering reduced minisuperspace models, different factor ordering have been chosen $[10-12,18,25])$.

Using the triad ADM canonical formulation, Bianchi models in pure $N=1$ supergravity have been studied in ref $[2-8,22,23,24]$. The quantum states may be described by a wave function of the form $\Psi\left(e_{A A^{\prime} i}, \psi_{A i}\right)$ where $e_{A A^{\prime} i}$ and $\psi_{A i}$ denote, respectively, the two-component spinor form of the tetrad and the spin- $\frac{3}{2}$ gravitino field. The wave function may be then expanded in even powers of $\psi_{A i}$, symbolically represented by $\psi^{0}, \psi^{2}, \psi^{4}$ up to $\psi^{6}$ because of the anti-commutations relations of the six spatial components of the gravitino fields (see ref.[6,7,15,16,24] for more details).

The analysis of locally supersymetric Bianchi class A models also began to face some unexpected difficulties. Supersymmetry (as well as other considerations) forbids minisuperspace models of class $B$. Firstly, models without supermatter had (simple) solutions only in the empty $\psi^{0}$ (bosonic) and fermionic filled $\psi^{6}$ sectors. More precisely, the physical 
states in these sectors were, respectively, given by (cf. ref. [6-8,13,16,22,23,24])

$$
\begin{gathered}
\psi^{0} \rightarrow e^{m^{p q} h_{p q}}, \\
\psi^{6} \rightarrow h e^{-m^{p q} h_{p q}} \prod_{i}\left(\psi_{i}^{A}\right)^{2} .
\end{gathered}
$$

Here $h$ is the determinant of the 3 -metric $h_{p q}$ and $m^{p q}$ is defined from the relation

$$
d w^{p}=\frac{1}{2} m^{p q} h^{-\frac{1}{2}} \epsilon_{q r s} \omega^{r} \otimes \omega^{s}
$$

where $\omega^{r}$ are basis of left-invariant 1-forms on the space-like hypersurface of homogeneity; the constant symmetric matrix $m^{p q}$ is fixed by the chosen Bianchi type (cf. e.g., [44]). Secondly, these states could be interpreted either as Hartle-Hawking no-boundary solutions [45] or wormhole states [46]. However, one could not found both of them in the same spectrum of solutions. According to different homogeneity conditions for the gravitino field (cf. ref. [8]), we either could find the Hartle-Hawking or the wormhole state. In addition, these solutions in minisuperspace were shown not to have any counterpart in the full theory because no states with zero (bosonic) or finite number of fermions are possible there $[19,20]$. Finally, when a cosmological constant was added no physical states were found $[14,15,16,36]$ (regarding the $\mathrm{k}=1$ Friedmann-Robertson-Walker model, where the fermionic degrees of freedom of the gravitino field are very restricted, a bosonic quantum physical state was found, namely the Hartle-Hawking solution [45] for a De Sitter state). It seemed that the gravitational and gravitino modes that were allowed to be excited in each supersymmetric Bianchi model contribute in such a way as to give only very simple states or even forbid any physical solutions of the quantum constraints.

All these results seemed difficult to accomodate. In fact, doubts were then raised in ref.[23,24]: even though canonical quantum supergravity has more constraints than ordinary quantum gravity, it has surely much more degrees of freedom than gravity ${ }^{1}$. Hence, why should we experience problems such as the above expressed, like few or even

1 One should also stress that the action of the full supergravity theory with boundary terms [1] is not fully invariant under supersymmetry transformations. The invariance of an action under the corresponding symmetries of the problem in study is an obvious desideratum [47]. In ref. [22] such invariance for supersymmetry transformations was achieved for the case of Bianchi class A models using appropriate extra boundary terms. 
no physical states in acceptable physical situations? The cause for the apparent paradoxical results mentioned in the previous paragraph was the use of an Ansatz too special for the $\psi^{2}$ and $\psi^{4}$ fermionic (middle) sectors in the wave function. This Ansatz [6-8,13-16,26] for Lorentz invariant fermionic sectors allowed only for two bosonic amplitudes in each of the $\psi^{2}$ and $\psi^{4}$ middle fermionic sectors. These were constructed only from the Lorentz irreducible modes of the gravitino field and corresponded to bilinear and quadratic terms in the gravitino field. However, there can be actually up to 15 such invariants, when we consider the Lorentz irreducible modes of the gravitational degrees of freedom as well (see $[23,24]$ for more details $)^{2}$. These 15 Lorentz invariant components for each fermionic middle sector correspond to a single one which satisfy a Wheeler-DeWitt type equation. For a particular factor ordering, we obtain the wormhole state in the bosonic sector and the Hartle-Hawking solution in the quartic fermionic sector. The extension of ref. [23,24] framework to Bianchi models with a cosmological constant term is currently under way [49].

A subsequent step would be to consider more general supergravity models involving lower-spin fields. One possibility is to take higher- $N$ gauged supergravity models [50]. Generically, these are technically difficult in the approach used in [1] because they may contain a $\Lambda$-term which breaks chirality. Some results on the canonical quantization of $d=4, N=2$ supergravity with a non-zero cosmological constant using Ashtekar variables was presented in [40]. However, the simple $N=2$ supergravity with a global $O(2)$ or $S O(3)$ symmetry $[50,51,52]$ may still prove to be useful within the ADM approach. In these particular cases, we do not have the imposition of a cosmological constant term. An analysis for the case of a Bianchi I model is currently in progress [53]. Another possibility is to consider the theory of $N=1$ supergravity coupled to supermatter, and in particular its supersymmetry constraints (see e.g., ref. [54]). Its canonical formulation can be found in refs. $[17,18]$.

Clearly, a richer and more interesting class of models is given by coupling supermatter to $N=1$ supergravity in 4 dimensions. In particular, a dimensional reduction allows one to obtain a $(1+0)$-dimensional theory with $\mathrm{N}=4$ supersymmetry from $(1+3)$ dimensional

\footnotetext{
2 For the case of a FRW model without supermatter and due to the restriction of the gravitino field to its spin- $\frac{1}{2}$ mode component, the "old" Ansatz for the wave function remains valid [48].
} 
(pure) $\mathrm{N}=1$ supergravity $[9,10,12]$. Although such minisuperspace constructions may provide us with further understanding of some specific aspects which we hope that will hold in some limit for the general 4-dimensional theory, one should also point out their limitations. The truncation of the inhomogenous modes constitute a severe restriction, in particulary in a Friedmann model where the anisotropy degrees of freedom have been frozen as well. The validity of the minisuperspace approximation in locally supersymmetric models is yet an open problem. Nevertheless, simplified models like the special case of a FRW universe may allow us to obtain interesting properties even if the results may strongly depend on the minisuperspace truncations. In ref.[9-12] an Ansatz for the gravitational and spin- $\frac{3}{2}$ fields was introduced in order to reduce pure $N=1$ supergravity in 4 dimensions to a locally supersymmetric quantum cosmological model in 1 dimension, assuming a Friedmann $k=+1$ geometry and homogeneity of the spin- $\frac{3}{2}$ field on the $S^{3}$ spatial sections. The Hamiltonian structure of the resulting theory was found, leading to the quantum constraint equations. The general solution to the quantum constraints is very simple in this case, and the Hartle-Hawking wave-function can be found. Following the particular supermatter model described in ref. [55], a FRW minisuperspace in $\mathrm{N}=1$ supergravity coupled to locally supersymmetric supermatter (a massive complex scalar with spin- $\frac{1}{2}$ partner) was considered in ref. $[10,11,12]$. In the massless case [11] a ground quantum wormhole state can be found as a solution of the quantum constraints in the form of an integral expression.

Locally supersymmetric quantum cosmological models obtained from the more general $\mathrm{N}=1$ supergravity theory in the presence of supermatter were instead considered in ref. $[18,21,25,26]$. Such theory is described in detail in ref. [54]. The theory was restricted in ref. $[18,21]$ to the special case of a $k=1$ FRW minisuperspace model with a family of complex spin-0 scalar fields together with their odd (anti-commuting) spin- $\frac{1}{2}$ partners. The Ansatz for the wave function of the universe was still found in the "old" approach. For the two-dimensional spherically symmetric and flat Kähler geometries new solutions with a simple form for the quantum states were found. In particular, the Hartle-Hawking solution is present but the wormhole state seems absent ${ }^{3}$. Furthermore, different factor

\footnotetext{
3 This issue has been recently addressed in ref.[62]. The differences in ref. [11,18,21] relatively to the presence
} or not of wormhole states can be seen as a consequence of two separate causes. On the one hand, the choice of Lagrange multipliers (which may simplify the form of the contraints and corresponding algebra) and on the other 
ordering were also considered. In ref. [25] a supermultiplet constituted by spin-1 $S U(2)$ gauge fields and their fermionic partners was added as well to the supermatter fields. By imposing the supersymmetry and Lorentz constraints it was found that no physical states were allowed ${ }^{4}$.

In this paper we will study instead a locally supersymmetric (diagonal) Bianchi typeIX model coupled to a scalar supermultiplet, formed by a complex spin-0 scalar fields together with their odd (anti-commuting) spin- $\frac{1}{2}$ partners. We choose the corresponding Kähler geometry to be a flat one. This Bianchi type-IX supersymmetric model will bear important differences as far as FRW models in $\mathrm{N}=1$ supergravity with supermatter are concerned.

Firstly, anisotropic gravitational degrees of freedom are now present. Consequently, the gravitino fields are no longer required to be severly restricted to their spin- $\frac{1}{2}$ modes (see ref. $[9-12,18,21,25])$ and hence the spin- $\frac{3}{2}$ modes will play an important role as we will see. In such a way we hope our minisuperspace model with supermatter may be able, in spite of the inhomogenous modes truncation, to better reveal some of the features of the full theory of $\mathrm{N}=1$ supergravity theory with supermatter.

Secondly, we will take a different approach from the one involving a direct dimensional reduction of the $d=4$ theory. Dimensional reduced one-dimensional models which inherits invariance under local time translations, Lorentz and supersymmetry transformations from 4-dimensional ones can be obtained by studying the (more complicated) non-diagonal Bianchi cases $[6,7,15]$. To obtain an Ansatz invariant under supersymmetry transfomations one must use a non-diagonal triad $e_{i}^{a}=b_{b}^{a} E_{i}^{b}$ where $b_{a b}$ is symmetric $(a, b=1,2,3)$ and combine it with a homogeneous spatial coordinate transformation and local Lorentz rotations. Such an approach was done in ref. [2-5,6] and was recently extended for the case of a Bianchi-I model with a scalar multiplet coupling [26]. However, it has also been

hand how we deal with the fermionic derivative ordering. A suitable combination of these two aspects seems to make a difference.

4 A possible reason could either be the use of the "old" type of Ansatz for the wave function of the universe or that the Ansätze for the spin-1 field and corresponding supersymmetric partner were not the more general one under supersymmetry transformations. In fact, an improved Ansatz for the Yang-Mills fields seems to allow physical states to be present in a locally supersymmetric FRW model with spin-1 fields [63]. 
pointed that the dimensional reduction with diagonal Bianchi models may be safely used $[5,56,57]$ as in the end the algebra of constraints is closed and consistently a supersymmetric one. Alternatively, the differential equations for our case study may be obtained instead by studying the quantum constraints of the full theory of $N=1$ supergravity with supermatter $[17,18,54]$ subject to a Bianchi type-IX (diagonal) ansatz for the tetrad and correspondingly to the gravitino fields. Our purposes in applying the quantum constraints of the full theory are, on the one hand, to make a full use of the recently obtained canonical formulation of $\mathrm{N}=1$ supergravity with supermatter [17], and on the other hand, to use the (simpler) diagonal Bianchi-IX metric. This is a valued approach since the relevant constraints are of first order in bosonic derivatives, unlike the case in quantized general relativity. We will then get expressions for the quantity $\delta \Psi / \delta e_{A A^{\prime}}^{i}$ which, when evaluated at a Bianchi metric, give the infinitesimal change of the wave function under a variation of the three scale factors of the Bianchi type-IX metric and hence allow the evolution of the wave function subject to the above mentioned Ansatz to be determined. The outcome will then be similar to the one obtained through a dimensional reduction. We would also like to stress a particular advantage of using the (diagonal) Bianchi IX model as opposed to more simple cases such as a (general) Bianchi-I. In the former, we will have non-zero gravitational potentials while in the later those will be zero. Hence, for the case of free matter one gets a model without self interactions for the Bianchi-I while in the Bianchi-IX case one expects a behaviour which will be, up to some extent, more realistic as far as the full theory is concerned.

Finally, we will analyse our Bianchi-IX supersymmetric model with supermatter accordingly to two cases: with and without a scalar field dependent analytical potential $P(\Phi)$ in the Lagrangian. The fact that we will have all the homogenous mode components of the gravitino field as well as the terms in the action corresponding to a gravitational potential may allow future research to address supersymmetry breaking features from other points of view. Indeed, supersymmetry breaking phenomena is related to the behaviour of the scalar field dependent analytical potential (cf. ref. [54]). Moreover, the presence of the analytical potential in the supersymetry constraints is similar in some sense to the one induced by a cosmological constant, therefore allowing for chirality to be broken. It will be interesting to check in the case $P(\Phi) \neq 0$ if any quantum-physical states are possible. Nevertheless, one should be very careful as to properly interpretate the results with respect 
to the choice of wave function Ansatz.

In fact, let us point out before proceeding that we will follow in this paper an Ansatz for the expansion of the wave function in fermionic sectors, which is still obtained within the "old" approach. Basically, we consider the simplest Lorentz invariants, constructed only from the irreducible spin- $\frac{1}{2}$ and $\frac{3}{2}$ components of the fermionic fields in presence (see eq.(3.13)). Obviously, we are aware that this is an overly restricted Ansatz as compared with the "new" approach developed in [23,24]. Our reasons are as follows.

On the one hand, the so called "new" approach conveys any final calculations of the explicit analytical solutions of the bosonic amplitudes for the fermionic middle sectors to a (second order) Wheeler-DeWitt type equation. It would be much harder to try to solve a Wheeler-DeWitt type equation for a Bianchi type-IX model with a complex scalar matter field, which to our knowledge do not exist yet in the literature (a study of a Bianchi-IX model with a real scalar field can be found in [62]). Moreover, the amplitudes of the bottom (bosonic) and top (fermionic filled) sectors in [23,24] are present in the "old" Ansatz procedure, using the (first order) differential equations directly obtained from the Lorentz and supersymmetry constraints. Furthermore, the middle sector solution obtained in [23] from the Wheeler-DeWitt type equation is not new in the sense that it was allready found out using a specific definition of homogeneity conditions for the gravitinos [8] and in the context of the "old" Ansatz construction.

On the other hand, we would like to obtain new solutions which would bear any physical significance with regard to minisuperspace Bianchi-IX cosmologies. A straightforward (although tedious) way would be to consider the (first order) set of differential equations obtained from the supersymmetric constraints, as the "old" procedure allows. We hope in this way to get solutions that ought to be present as well in the approach of $[23,24]$, possibly giving us an insight on how to generalize it to couplings with supermatter. As we will see, solutions in the top and bottom sectors as well as in some middle sectors are obtained for our model when the analytical potential is zero. We suspect that these or similar properties and solutions would be obtained in the "new" approach but at the expense of having to deal with a Wheeler-DeWitt equation. Of course, we could possibly find out other solutions with a more general Lorentz invariant Ansatz for the wave function of the universe. Our point is that the "old" Ansatz construction may still be usefull, up 
to a certain point, if addressed within its proper context.

The above statements should however be taken with care as far as the inclusion of chirality breaking terms (like a cosmological constant) are concerned. The non-existence of physical states in that case is again another indication that the "old" restrictive Ansatz is not adequate when dealing with these type of situations. Surely the "new" approach is a welcomed feature for the theory of canonical quantization of locally supersymmetric models.

This paper is then organized as follows. In section II we briefly describe the canonical formulation of $\mathrm{N}=1$ supergravity with supermatter, following ref. [17,18]. For the sake of completeness we present the general theory with gauge supermatter, ie, with a family of spin-0 and 1 fields and their fermionic partners. Some basic features are improved as well. More precisely, the variation of some physical observables under supersymmetry transformations obtained from the supersymmetry constraints generators in $[17,18]$ do not coincide with the ones present in the literature (see, e.g. ref. [54]) for the cases of the spin$\frac{1}{2}$ partner of the scalar field and the gravitino. We explain why this problem was present and suggest the inclusion of adequate terms in the Hamiltonian formulation as to get consistent results with the usual supersymmetry transformations. The quantization of the diagonal Bianchi-IX model is studied in section III, by studying the quantum constraints in the full theory subject to a suitable Bianchi-IX Ansätze for the fields. The chosen supermatter model will be restricted to a spin-0 and spin- $\frac{1}{2}$ supermultiplet, corresponding to a 2-dimensional flat Kähler geometery. We will discuss separatly two cases: when the scalar field dependent analytic potential $P(\Phi)$ is arbitrarly and when $P(\Phi)$ is zero. For our Lorentz invariant Ansatz of the wave function of the universe we will find that in the later the wave function has a simple form. Namely, the only non zero components of the wave function can be found in the sectors with no fermions (bosonic) and in three other sectors, more precisely filled with just the spin- $\frac{1}{2}$ fermionic partners of the scalar field, another filled with just the spin- $\frac{1}{2}$ and $\frac{3}{2}$ mode components of the spatially homogeneous gravitino field and finally one totally filled with spin- $\frac{1}{2}$ fermionic partners of the scalar field as well as the the spin- $\frac{1}{2}$ and $\frac{3}{2}$ mode components of the gravitino field. In section IV we present our discussons and conclusions. 


\section{Canonical formulation of $\mathrm{N}=1$ supergravity with supermatter}

The Lagrangian of the more general theory of $\mathrm{N}=1$ supergravity with supermatter is given in eq. (25.12) of [54] and it is too long to write out here. It depends on the tetrad $e^{A A^{\prime}}{ }_{\mu}$, where $A, A^{\prime}$ are two-component indices using the conventions of [1] and $\mu$ is a space-time index, the spin- $\frac{3}{2}$ gravitino field $\left(\psi_{\mu}^{A}, \widetilde{\psi}^{A^{\prime}}\right)$. whose components are taken to be odd elements of a Grassmann algebra, on a vector field $A_{\mu}^{(a)}$ labelled by a group index $(a)$, its spin- $\frac{1}{2}$ partner $\left(\lambda_{A}^{(a)}, \widetilde{\lambda}_{A^{\prime}}^{(a)}\right)$, a family of scalars $\left(\Phi^{I}, \Phi^{J^{*}}\right)$ and their spin- $\frac{1}{2}$ partner $\left(\chi_{A}^{I}, \tilde{\chi}_{A^{\prime}}^{J^{*}}\right)$. The indices $I, \ldots, J^{*}, \ldots$ are Kähler indices, and there is a Kähler metric

$$
g_{I J^{*}}=K_{I J^{*}}
$$

on the space of $\left(\Phi^{I}, \Phi^{J^{*}}\right)$, where $K_{I J^{*}}$ is a shorthand for $\partial^{2} K / \partial \Phi^{I} \partial \Phi^{J^{*}}$ with $K$ the Kähler potential. Each index $(a)$ corresponds to an independent Killing vector field of the Kähler geometry. Such Killing vectors are holomorphic vector fields:

$$
\begin{aligned}
X^{(b)} & =X^{I(b)}\left(\Phi^{J}\right) \frac{\partial}{\partial \Phi^{I}}, \\
X^{*}(b) & =X^{I^{*}(b)}\left(\Phi^{J^{*}}\right) \frac{\partial}{\partial \Phi^{I^{*}}} .
\end{aligned}
$$

Killing's equation implies that there exist real scalar functions $D^{(a)}\left(\Phi^{I}, \Phi^{I^{*}}\right)$ known as Killing potentials, such that

$$
\begin{aligned}
g_{I J^{*}} X^{J^{*}(a)} & =i \frac{\partial}{\partial \Phi^{I}} D^{(a)} \\
g_{I J^{*}} X^{I(a)} & =-i \frac{\partial}{\partial \Phi^{J^{*}}} D^{(a)} .
\end{aligned}
$$

In the Hamiltonian decomposition, the variables are split into $e^{A A^{\prime}}{ }_{i}, \psi_{i}^{A}, \widetilde{\psi}_{i}^{A^{\prime}}, A_{i}^{(a)}$, $\left(i=1,2,3\right.$, denotes spatial components) $\lambda_{A}^{(a)}, \widetilde{\lambda}_{A^{\prime}}^{(a)}, \chi_{A}^{I}, \widetilde{\chi}_{A^{\prime}}^{J^{*}}, \Phi^{I}, \Phi^{J^{*}}$, which together with the bosonic momenta are the basic dynamical variables of the theory, and the Lagrange multipliers $N, N^{i}, \psi_{0}^{A}, \widetilde{\psi}_{0}^{A^{\prime}}, A_{0}^{(a)}, M_{A B}, \widetilde{M}_{A^{\prime} B^{\prime}}$, where $N, N^{i}$ are formed from the $e^{A A^{\prime}}{ }_{0}$ and the $e^{A A^{\prime}}{ }_{i}[1]$, and $M_{A B}, \widetilde{M}_{A^{\prime} B^{\prime}}$ involve the zero components $\omega_{A B 0}, \widetilde{\omega}_{A^{\prime} B^{\prime} 0}$ of the connection (see below). The constraint generators are functions of the basic dynamical variables. 
The total Hamiltonian has the form

$$
\begin{aligned}
H= & N \mathcal{H}_{\perp}+N^{i} \mathcal{H}_{i}+\psi_{0}^{A} S_{A}+\widetilde{S}_{A^{\prime}} \widetilde{\psi}_{0}^{A^{\prime}} \\
& +A_{0}^{(a)} Q_{(a)}+M_{A B} J^{A B}+\widetilde{M}_{A^{\prime} B^{\prime}} \widetilde{J}^{A^{\prime} B^{\prime}}
\end{aligned}
$$

expected for a theory with the corresponding gauge invariances. Here $N$ and $N^{i}$ are the lapse function and shift vector, while $\mathcal{H}_{\perp}$ and $\mathcal{H}_{i}$ are the (modified) generators of deformations in the normal and tangential directions. $S_{A}$ and $\widetilde{S}_{A^{\prime}}$ are the local supersymmetry generators, $Q_{(a)}$ is the generator of gauge invariance, and $J^{A B}$ and $\widetilde{J}^{A^{\prime} B^{\prime}}$ are the generators of local Lorentz rotations. Classically, all the constraints $\mathcal{H}_{\perp}, \mathcal{H}_{i}, S_{A}, \widetilde{S}_{A^{\prime}}, Q_{(a)}, J^{A B}, J^{A^{\prime} B^{\prime}}$ vanish, and form a set of (first-class) constraints, satisfying an algebra. Quantum mechanically, the constraints become operators which annihilate physical states $\Psi$ :

$$
\begin{aligned}
\mathcal{H}_{\perp} \Psi=0, & \mathcal{H}_{i} \Psi=0, \quad S_{A} \Psi=0, \quad \bar{S}_{A^{\prime}} \Psi=0, \\
Q_{(a)} \Psi=0, & J^{A B} \Psi=0, \quad \bar{J}^{A^{\prime} B^{\prime}} \Psi=0 .
\end{aligned}
$$

For the gravitino and spin- $\frac{1}{2}$ fields, the canonical momenta give second-class constraints of the types described in $[1,43,58]$. These are eliminated when Dirac brackets are introduced $[1,10,43]$ instead of the original Poisson brackets. In particular, one obtains nontrivial Dirac brackets for $p_{A A^{\prime}}{ }^{i}$, (the momentum conjugate to $e^{A A^{\prime}}{ }_{i}$ ), for $\psi_{i}^{A}$ and $\widetilde{\psi}^{A^{\prime}}$, for $\lambda_{A}^{(a)}$ and $\tilde{\lambda}_{A^{\prime}}^{(a)}$, for $\chi_{A}^{I}$ and $\widetilde{\chi}_{A^{\prime}}^{J^{*}}$, and for $\pi_{L}, \pi_{L^{*}}$, (the momenta conjugate to $\Phi^{L}, \Phi^{L^{*}}$ ). These can be made into simple brackets as follows.

The brackets involving $p_{A A^{\prime}}{ }^{i}, \psi^{A}{ }_{i}$ and $\widetilde{\psi}^{A^{\prime}}$ can be simplified as in the case of pure $N=1$ supergravity [1] by redefining

$$
p_{A A^{\prime}}^{i} \rightarrow \hat{p}_{A A^{\prime}}^{i}=p_{A A^{\prime}}{ }^{i}-\frac{1}{\sqrt{2}} \epsilon^{i j k} \psi_{A j} \widetilde{\psi}_{A^{\prime} k} .
$$

The $\Phi^{K}$ and $\Phi^{K^{*}}$ dependence of $K_{I J^{*}}$ is responsible for unwanted Dirac brackets among $\chi_{A}^{I}, \widetilde{\chi}_{A}^{J^{*}}, \pi_{L}$ and $\pi_{L^{*}}$. In fact, defining $\pi_{I A}$ and $\widetilde{\pi}_{I^{*} A^{\prime}}$ to be the momenta conjugate to $\chi^{I A}$, and $\widetilde{\chi}^{I^{*} A^{\prime}}$, respectively, one has

$$
\begin{aligned}
& \pi_{I A}+\frac{i h^{\frac{1}{2}}}{\sqrt{2}} K_{I J^{*} n_{A A^{\prime}}} \tilde{\chi}^{J^{*} A^{\prime}}=0, \\
& \widetilde{\pi}_{J^{*} A^{\prime}}+\frac{i h^{\frac{1}{2}}}{\sqrt{2}} K_{I J^{*}} n_{A A^{\prime}} \chi^{I A}=0 .
\end{aligned}
$$


Here $n^{A A^{\prime}}$ is the spinor version of the unit future-directed normal vector $n^{\mu}$, obeying

$$
n_{A A^{\prime}} n^{A A^{\prime}}=1, \quad n_{A A^{\prime}} e^{A A^{\prime}}=0 .
$$

One cures this by introducing the modified variables

$$
\begin{gathered}
\hat{\chi}_{I A}=h^{\frac{1}{4}} K_{I J^{*}}^{\frac{1}{2}} \delta^{K J^{*}} \chi_{K A}, \\
\hat{\tilde{\chi}}_{I^{*} A^{\prime}}=h^{\frac{1}{4}} K_{J I^{*}}^{\frac{1}{2}} \delta^{J K^{*}} \widetilde{\chi}_{K^{*} A^{\prime}},
\end{gathered}
$$

where the factor of $h^{1 / 4}$ has been introduced for later use (in the time gauge - see below). $K_{I J^{*}}^{\frac{1}{2}}$ denotes a "square root" of the Kähler metric, obeying

$$
K_{I J^{*}}^{\frac{1}{2}} \delta^{K J^{*}} K_{K L^{*}}^{\frac{1}{2}}=K_{I L^{*}}
$$

This may be found by diagonalizing $K_{I J^{*}}$ via a unitary transformation, assuming that the eigenvalues are all positive. One needs to assume that there is an "identity metric" $\delta^{K J^{*}}$ defined over the Kähler manifold; this will be true if a positive-definite vielbein field can be introduced. Then the second-class constraints in Eq. (2.7) read

$$
\begin{aligned}
\hat{\pi}_{I A}+\frac{i}{\sqrt{2}} \delta_{I J^{*}} n_{A A^{\prime}} \hat{\tilde{\chi}}^{J^{*} A^{\prime}}=0, \\
\hat{\tilde{\pi}}_{I^{*} A^{\prime}}+\frac{i}{\sqrt{2}} \delta_{I J^{*}} n_{A A^{\prime}} \hat{\chi}^{J A}=0 .
\end{aligned}
$$

Finally, the brackets among $\hat{p}_{A A^{\prime}}{ }^{i}, \lambda_{A}^{(a)}, \widetilde{\lambda}_{A^{\prime}}^{(a)}, \hat{\chi}_{A}^{I}$ and $\hat{\tilde{\chi}}_{A^{\prime}}^{J^{*}}$ are dealt with by defining (see ref. $[58,59])$

$$
\hat{\lambda}_{A}^{(a)}=h^{\frac{1}{4}} \lambda_{A}^{(a)}, \quad \hat{\tilde{\lambda}}_{A^{\prime}}^{(a)}=h^{\frac{1}{4}} \widetilde{\lambda}_{A^{\prime}}^{(a)},
$$

and then going to the time gauge. In this case, the tetrad component $n^{a}$ of the normal vector $n^{\mu}$ is henceforward restricted by

$$
n^{a}=\delta_{0}^{a},
$$

or equivalently

$$
e^{0}{ }_{i}=0
$$


Thus the original Lorentz rotation freedom becomes replaced by that of spatial rotations. In the time gauge, the geometry is described by the triad $e^{\alpha}{ }_{i}(\alpha=1,2,3)$, and the conjugate momentum ${ }^{3}$ is $p_{\alpha}{ }^{i}$.

The resulting Dirac brackets are

$$
\begin{aligned}
& {\left[p_{\alpha}{ }^{i}, \psi^{B}{ }_{j}\right]_{D}=0,} \\
& {\left[p_{\alpha}{ }^{i}, \widetilde{\psi}^{B^{\prime}}\right]_{D}=0,} \\
& {\left[p_{\alpha}{ }^{i}, \hat{p}_{\beta}{ }^{j}\right]_{D}=0,} \\
& {\left[p_{\alpha}{ }^{i}, \hat{\lambda}_{A}^{(a)}\right]_{D}=0,} \\
& {\left[p_{\alpha}{ }^{i}, \hat{\chi}^{I}{ }_{A}\right]_{D}=0, \text { etc. }} \\
& {\left[\pi_{L}, \pi_{M}\right]_{D}=0,} \\
& {\left[\pi_{L}, \hat{\chi}^{A}\right]_{D}=0, \text { etc. }}
\end{aligned}
$$

The remaining brackets are standard; the nonzero fermionic brackets are

$$
\begin{gathered}
{\left[\hat{\lambda}_{A}^{(a)}(x), \hat{\tilde{\lambda}}_{A^{\prime}}^{(b)}(x)\right]_{D}=\sqrt{2} i n_{A A^{\prime}} \delta^{(a)(b)} \delta\left(x, x^{\prime}\right),} \\
{\left[\hat{\chi}_{A}^{I}(x), \hat{\tilde{\chi}}_{A^{\prime}}^{J^{*}}\left(x^{\prime}\right)\right]_{D}=\sqrt{2} i n_{A A^{\prime}} \delta^{I J^{*}} \delta\left(x, x^{\prime}\right),} \\
{\left[\psi_{i}^{A}(x), \widetilde{\psi}^{A^{\prime}}{ }_{j}\left(x^{\prime}\right)\right]_{D}=\frac{1}{\sqrt{2}} D^{A A^{\prime}}{ }_{i j} \delta\left(x, x^{\prime}\right),}
\end{gathered}
$$

where

$$
D^{A A^{\prime}}{ }_{i j}=-2 i h^{-\frac{1}{2}} e^{A B^{\prime}} e_{B B^{\prime} i} n^{B A^{\prime}}
$$

We would like to point out that some of the signs and factors in the above expressions are different from the ones in ref. [1]. The quantum representation of momenta and coordinate will be done accordingly, in order to achieve self-consistency (see section III).

Let us now address the supersymmetry constraints from the Hamiltonian formalism. We will follow closely the framework presented in ref.[1,43] and adequality extended it to

3 Notice that $\pi^{i j} \equiv-\frac{1}{2} p^{(i j)}=\frac{1}{2} e^{A A^{\prime}(i} p_{A A^{\prime}}^{j)}=-\frac{1}{2} e^{\alpha(i} p_{\alpha}^{j)}$ where the last equality follows from the time gauge conditions; see ref. [43,58]. 
the case where gauged supermatter is present. As stated above, the momenta conjugate to the basic fields are found from the Lagrangian and leading to a number of primary and secondary constraints, these last characteristic of systems with fermions. Eliminating these and obtaining modified bracket relations, as explained, we obtain an explicit form for the terms present in expression (2.4). However, the full Hamiltonian (2.4) contains arbitrary Lorentz rotations and these ought to be included through a geometrically meaningfull Lagrangian multipliers, namely the time component $\omega_{A B 0}, \tilde{\omega}_{A^{\prime} B^{\prime} 0}$ of the connection forms multiplied by a minus sign $[1,43]$. Choosing to implement this procedure, the improved constraints in (2.4) will then differ from the previous ones by terms proportional to projections of $J_{A B}$ or its derivatives. The parts in $J_{A B}, \widetilde{J}_{A^{\prime} B^{\prime}}$ corresponding to the spin-2 and $\frac{3}{2}$ fields will contribute to $S_{A}, \bar{S}_{A^{\prime}}$ as in [1,43], after expanding the spatial covariant derivative in its torsion-free and contorsion parts. The inclusion of components from $J_{A B}$ which depend on the mater fields will contribute with new terms of the type $\psi \chi \bar{\chi}, \quad \psi \lambda \bar{\lambda}$ and their hermitian conjugates to the supersymmetry constraints [43]. We notice that this last step is missing in the procedure employed in ref. [17]. In the end of this section we further discuss the implications of its absence and which problems its presence might solve. The supersymmetry constraint $\widetilde{S}_{A^{\prime}}$ is then found to be

$$
\begin{aligned}
& \widetilde{S}_{A^{\prime}}=-\sqrt{2} i e_{A A^{\prime} i} \psi^{A}{ }_{j} \pi^{i j}+\sqrt{2} \epsilon^{i j k} e_{A A^{\prime} i}{ }^{3 s} \widetilde{D}_{j} \psi_{k}^{A}
\end{aligned}
$$

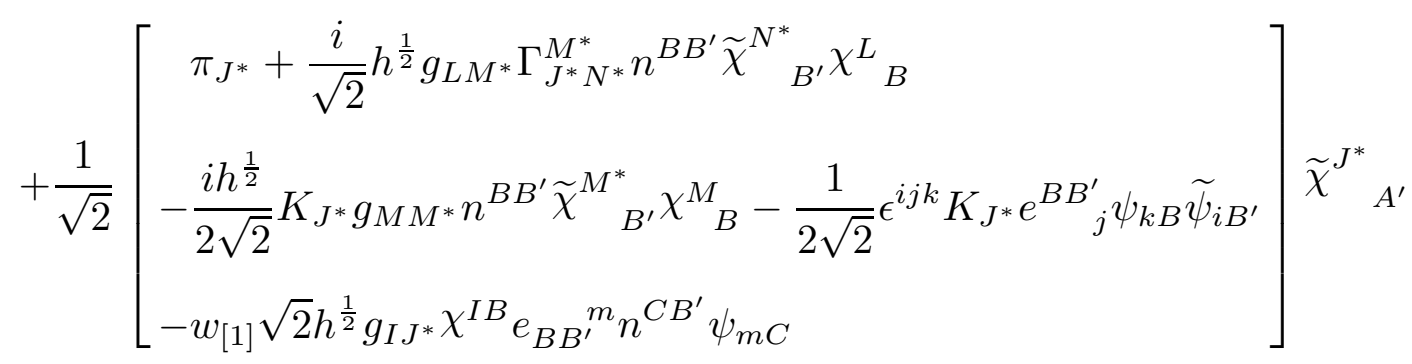

$$
\begin{aligned}
& -\sqrt{2} h^{\frac{1}{2}} g_{I J^{*}}\left(\widetilde{\mathcal{D}}_{i} \Phi^{I}\right) \widetilde{\chi}_{B^{\prime}}^{J^{*}} n^{B B^{\prime}} e_{B A^{\prime}}{ }^{i}+w_{[2]} \frac{i}{2} g_{I J *} \epsilon^{i j k} e_{A A^{\prime} j} \psi^{A}{ }_{i} \widetilde{\chi}^{J^{*} B^{\prime}} e_{B B^{\prime} k} \chi^{I B} \\
& +w_{[3]} \frac{1}{4} h^{\frac{1}{2}} \psi_{A i}\left(e_{B A^{\prime}}^{i} n^{A C^{\prime}}-e^{A C^{\prime} i} n_{B A^{\prime}}\right) g_{I J^{*}} \widetilde{\chi}_{C^{\prime}}^{J^{*}} \chi^{I B} \\
& -h^{\frac{1}{2}} \exp (K / 2)\left[2 P n^{A}{ }_{A^{\prime}} e_{A B^{\prime}}{ }^{i} \widetilde{\psi}_{i}^{B^{\prime}}+i\left(D_{I} P\right) n_{A A^{\prime}} \chi^{I A}\right] \\
& -\frac{i}{\sqrt{2}} \pi^{n(a)} e_{B A^{\prime} n} \lambda^{(a) B}+\frac{1}{2 \sqrt{2}} \epsilon^{i j k} e_{B A^{\prime} k} \lambda^{(a) B} F_{i j}^{(a)}+\frac{1}{\sqrt{2}} h^{\frac{1}{2}} g D^{(a)} n_{A^{\prime}}^{A} \lambda_{A}^{(a)}
\end{aligned}
$$




$$
\begin{aligned}
& +w_{[4]} \frac{1}{4} h^{\frac{1}{2}} \psi_{A i}\left(e_{B A^{\prime}}{ }^{i} n^{A C^{\prime}}-e^{A C^{\prime} i} n_{B A^{\prime}}\right) \widetilde{\lambda}_{C^{\prime}}^{(a)} \lambda^{(a) B} \\
& -\frac{i}{4} h^{\frac{1}{2}} n^{B B^{\prime}} \lambda^{(a)}{ }_{B} \widetilde{\lambda}_{B^{\prime}}^{(a)} K_{J^{*}} \widetilde{\chi}^{J^{*}}{ }_{A^{\prime}}
\end{aligned}
$$

where $\lambda_{A}^{(a)}, \tilde{\lambda}_{A^{\prime}}^{(a)}$ and $\chi_{I A}, \tilde{\chi}_{I^{*} A^{\prime}}$ should be redefined as in Eqs. (2.9), (2.12). The other supersymmetry constraint $\left(S_{A}\right)$ is just the hermitian conjugate of $(2.21)$. Here $e_{A A^{\prime} i}=$ $\sigma_{A A^{\prime}}^{\alpha} e_{\alpha i}[1]$, where $\sigma_{A A^{\prime}}^{\alpha}(\alpha=1,2,3)$ are Infeld-van der Waerden symbols and $\pi^{i j}=$ $-\frac{1}{2} e^{\alpha(i} \hat{p}_{\alpha}{ }^{j)}$. Notice that the three last lines in (2.21) correspond to the presence of a spin-1 field and fermionic partner in the supermatter content, i.e., if gauged supermatter is considered [54,60]. These terms will not be used in our Bianchi type-IX model. The $w_{[i]}, i=1,2,3,4$ denote numerical coefficients which correspond to the inclusion of the terms $\psi \chi \bar{\chi}, \quad \psi \lambda \bar{\lambda}$ and their hermitian conjugates to the supersymmetry constraints via $\omega_{A B 0} J^{A B}$ and hermitian conjugate. Also [54]

$$
\begin{aligned}
{ }^{3 s} \widetilde{\mathcal{D}}_{j} \psi_{k}^{A} & =\partial_{j} \psi^{A}{ }_{k}+{ }^{3 s} \omega^{A}{ }_{B j} \psi_{k}^{B} \\
& +\frac{1}{4}\left(K_{K} \widetilde{\mathcal{D}}_{j} \Phi^{K}-K_{K^{*}} \widetilde{\mathcal{D}}_{j} \Phi^{K^{*}}\right) \psi_{k}^{A} \\
& +\frac{1}{2} g A_{j}^{(a)}\left(\operatorname{Im} F^{(a)}\right) \psi_{k}^{A},
\end{aligned}
$$

where ${ }^{3 s} \omega_{A B j},{ }^{3 s} \widetilde{\omega}_{A^{\prime} B^{\prime} j}$ give the torsion-free three-dimensional connection, and

$$
\widetilde{\mathcal{D}}_{i} A^{K}=\partial_{i} A^{K}-g A_{i}^{(a)} X^{K(a)}
$$

with $g$ the gauge coupling constant and $X^{K(a)}$ the ath Killing vector field. Furthermore, the analytic functions $F^{(a)}\left(\Phi^{J}\right)$ and $F^{*}(a)\left(\Phi^{I^{*}}\right)$ arise [54,60] from the transformation of the Kähler potential $K$ under an isometry generated by the Killing vectors $X^{(a)}$ and $X^{*}(a)$. Also, in Eq. $(2.21), \pi^{n(a)}$ is the momentum conjugate to $A_{n}^{(a)}, K_{J^{*}}$ denotes $\partial K / \partial \Phi^{J^{*}}, \Gamma_{J^{*} N^{*}}^{M^{*}}$ denotes the Christoffel symbols [54,60] of the Kähler geometry, and $P=P\left(\Phi^{I}\right)$ gives the anlytical potential of the theory.

The gauge generator $Q^{(a)}$ is given classically by

$$
\begin{aligned}
Q^{(a)} & =-\partial_{n} \pi^{n(a)}-g f^{a b c} \pi^{n(b)} A_{n}^{(c)} \\
& +g\left(\pi_{I} X^{I(a)}+\pi_{I^{*}} X^{I^{*}(a)}\right)
\end{aligned}
$$




$$
\begin{aligned}
& +\sqrt{2} i h^{\frac{1}{2}} g K_{M I^{*}} n^{A A^{\prime}} X^{J^{*}(a)} \Gamma_{J^{*} N^{*}}^{I^{*}} \tilde{\chi}_{A^{\prime}}^{N^{*}} \chi_{A}^{M} \\
& -\sqrt{2} i h^{\frac{1}{2}} g n^{A A^{\prime}} \widetilde{\lambda}_{A^{\prime}}^{(b)}\left[f^{a b c} \lambda_{A}^{(c)}+\frac{1}{2} i\left(\operatorname{Im} F^{(a)}\right) \lambda_{A}^{(b)}\right] \\
& +\sqrt{2} i h^{\frac{1}{2}} g n^{A A^{\prime}} K_{I J^{*}} \widetilde{\chi}_{A^{\prime}}^{J^{*}}\left[\frac{\partial X^{I(a)}}{\partial \Phi^{J}} \chi_{A}^{J}+\frac{1}{2} i\left(I m F^{(a)}\right) \chi_{A}^{I}\right] \\
& -\frac{i}{\sqrt{2}} g\left(\operatorname{Im} F^{(a)}\right) \epsilon^{i j k} \widetilde{\psi}_{i A^{\prime}} e^{A A^{\prime}}{ }_{j} \psi_{A k},
\end{aligned}
$$

where $f^{a b c}$ are the structure constants of the isometry group.

It is worthwhile to notice that we expect now to obtain the correct transformation properties (cf. ref. [54]) of the physical fields under both supersymmetry transformations, using brackets $\delta_{\xi} \psi_{i}^{A} \equiv\left[\widetilde{\xi}_{A^{\prime}} \widetilde{S}^{A^{\prime}}, \psi_{i}^{A}\right]_{D}$, etc, where $\xi$ is a constant spinor parametrizing the supersymmetric transformation. In fact, that was not possible for some fields, when using the explicit form for the supersymmetry constraints in ref. [17,18] as it can be checked. The reasons are as follows. On the one hand, the matter terms in the Lorentz constraints $J_{A B}, \widetilde{J}_{A^{\prime} B^{\prime}}$ were not included in the supersymmetry constraints, following and extending the framework presented in [43]. On the other hand, expressions only valid in pure $\mathrm{N}=1$ supergravity were employed to simplify the supersymmetry constraints with supermatter. Namely, the expressions for $S_{A}=0, \widetilde{S}_{A^{\prime}}=0$ in pure N=1 supergravity were used to rewrite the spatial covariant derivative ${ }^{3} D_{i}$ in terms of its torsion free part ${ }^{3 s} D_{i}$ and remaining terms which include the contorsion. When supermatter is present, we expect the different matter fields to play a role in the Lorentz constraints terms which ought to be included in the supersymmetry constraints once $\omega_{A B}^{0} J^{A B}$ and its hermitian conjugate are employed in the canonical action. A similar argument applies when we expand ${ }^{3} D_{i}$ but using (2.22), $(2.23)$.

\section{Quantization of the diagonal Bianchi type-IX model}

In this section we study the Bianchi type-IX model with spatial metric in diagonal form, using the supersymmetry constraints derived in the previous section. We restrict our case study to a supermatter model constituted only by a scalar field and its spin $-\frac{1}{2}$ partner with a two-dimensional flat Kähler geometry. The Kähler potential would be just $\phi \bar{\phi}$, the Kähler metric is $g_{\phi \bar{\phi}}=1$ and the Levi-Civita connections are zero. The scalar 
super-multiplet, consisting of a complex massive scalar field $\phi$ and massive spin- $\frac{1}{2}$ field $\chi_{A}, \bar{\chi}_{A^{\prime}}$ are chosen to be spatially homogeneous, depending only on time.

The 4-metric $g_{\mu \nu}$ of diagonal Bianchi type-IX is given by

$$
g_{\mu \nu}=\eta_{a b} e_{\mu}^{a} e_{\nu}^{b}
$$

where $\eta_{a b}$ is the Minkowski metric $(\mu, \nu=0, \ldots, 3 ; a, b=0, \ldots, 3)$ and the non-zero components of the tetrad $e^{a}{ }_{\mu}$ are given by

$$
\begin{aligned}
e_{0}^{0}=N, \quad e_{0}^{1} & =a_{1} N^{i} E_{i}^{1}, \quad e_{0}^{2}=a_{2} N^{i} E_{i}^{2}, \quad e_{0}^{3}=a_{3} N^{i} E_{i}^{3}, \\
e_{i}^{1} & =a_{1} E_{i}^{1}, \quad e_{i}^{2}=a_{2} E_{i}^{2}, \quad e_{i}^{3}=a_{3} E_{i}^{3} .
\end{aligned}
$$

Here $E_{i}^{1}, E_{i}^{2}, E_{3}^{3}(i=1,2,3)$ are a basis of unit left-invariant one-forms on the three-sphere [44] and $N, N^{i}, a_{1}, a_{2}, a_{3}$ are spatially constant. We can also write

$$
h_{i j}=a_{1}^{2} E_{i}^{1} E_{j}^{1}+a_{2}^{2} E_{i}^{2} E^{2}{ }_{j}+a_{3}^{2} E_{i}^{3} E^{3}{ }_{j} .
$$

In the calculation, we shall repeatedly need the expression:

$$
\begin{aligned}
\omega_{A B i} n^{A}{ }_{B^{\prime}} e^{B B^{\prime} j} & =\frac{i}{4}\left(\frac{a_{3}}{a_{1} a_{2}}+\frac{a_{2}}{a_{3} a_{1}}-\frac{a_{1}}{a_{2} a_{3}}\right) E_{i}^{1} E^{1 j} \\
& +\frac{i}{4}\left(\frac{a_{1}}{a_{2} a_{3}}+\frac{a_{3}}{a_{2} a_{1}}-\frac{a_{2}}{a_{3} a_{1}}\right) E_{i}^{2} E^{2 j} \\
& +\frac{i}{4}\left(\frac{a_{2}}{a_{3} a_{1}}+\frac{a_{1}}{a_{2} a_{3}}-\frac{a_{3}}{a_{1} a_{2}}\right) E_{i}^{3} E^{3 j} .
\end{aligned}
$$

We require that the components $\left(\psi_{0}^{A}, \bar{\psi}_{0}^{A^{\prime}}\right)$ be functions of time only. We further require that $\psi^{A}{ }_{i}$ and $\tilde{\psi}^{A^{\prime}}{ }_{i}$ be spatially homogeneous in the basis $e_{i}^{a}$.

We now proceed to solve the supersymetry and Lorentz constraints for the case of a diagonal Bianchi-IX model with a scalar supermultiplet. The $\mathcal{H}_{\perp}$ and $\mathcal{H}_{i}$ constraints can be defined through the anti-commutator of $S_{A}$ and $\bar{S}_{A^{\prime}}$, as in the case of $N=1$ supergravity without matter fields $[1,22,23,24]$. Thus the remaining constraints imply $\mathcal{H}_{\perp} \Psi=0, \mathcal{H}_{i} \Psi=0$; if one could find a solution of the remaining quantum constraints, the $\mathcal{H}_{\perp}$ and $\mathcal{H}_{i}$ constraints would follow (with a certain choice of factor-ordering). 
A quantum description can be made by studying (for example) Grassmann-algebravalued wave functions of the form $\Psi\left[e_{i}^{A A^{\prime}}, \psi_{i}^{A}, \bar{\chi}_{A}, \phi, \bar{\phi}\right]$. The choice of $\bar{\chi}_{A} \equiv n_{A} A^{\prime} \bar{\chi}_{A^{\prime}}$ rather than $\chi_{A}$ is designed so that the quantum constraint $\bar{S}_{A^{\prime}}$ should be of first order in momenta (cf. also ref. [9-12,18,21,25]). For simplicity, we have droped the hat "^" henceforth and apply our homogeneous Bianchi-IX Ansatze consistently throughout the paper.

The momenta are represented by

$$
\begin{aligned}
& p_{A A^{\prime}}{ }^{i} \rightarrow-i \hbar \frac{\delta}{\delta e^{A A^{\prime}}{ }_{i}}-\frac{1}{\sqrt{2}} \epsilon^{i j k} \psi_{A j} \bar{\psi}_{A^{\prime} k}, \\
& \pi_{\phi} \rightarrow-i \hbar \frac{\partial}{\partial \phi}, \\
& \pi_{\bar{\phi}} \rightarrow-i \hbar \frac{\partial}{\partial \bar{\phi}}, \\
& \bar{\psi}_{i}^{A^{\prime}} \rightarrow \frac{1}{\sqrt{2}} i \hbar D^{A A^{\prime}}{ }_{j i} h^{\frac{1}{2}} \frac{\partial}{\partial \psi^{A}{ }_{j}}, \\
& \chi^{A} \rightarrow-\sqrt{2} \hbar \frac{\partial}{\partial \bar{\chi}^{A}} .
\end{aligned}
$$

We have made the replacements $\delta \Psi / \delta \psi^{B}{ }_{j} \longrightarrow h^{\frac{1}{2}} \partial \Psi / \partial \psi^{B}{ }_{j}, \delta \Psi / \delta \bar{\chi}^{A} \rightarrow \partial \Psi / \partial \bar{\chi}^{A}$, where $\partial / \partial \psi_{j}^{A}, \partial / \partial \bar{\chi}^{A}$ denotes left differentiation. It is worthwhile to make the following comments about these replacements. The $h^{\frac{1}{2}}$ factor is necessary as to ensure that each term has the correct weight in the equations, namely when one takes a variation of a Bianchi geometry whose spatial sections are compact, multiplying by $\delta / \delta h_{i j}$ and integrating over the three-geometry $[6,7,14,15,16]$ : the cause can be identified in the term $h^{-\frac{1}{2}}$ in expression (2.20). One can check, e.g., that the inclusion of $h^{\frac{1}{2}}$ gives the correct supersymmetry constraints in the $k=+1$ Friedmann model, where the model was quantized using the alternative approach via a supersymmetric Ansatz [9-12,21,25]. It is interesting as well to notice that for the $\chi, \bar{\chi}$ fields no such requirements seemed to be needed to establish the equations for the bosonic amplitudes of the wave function of the universe.

The supersymmetry constraints become then, in differential operator form

$$
\bar{S}_{A^{\prime}}=-i \sqrt{2}\left[-i \hbar \frac{1}{4}\left[e_{A A^{\prime} i} \psi_{j}^{A}\right] e^{C C^{\prime} i} \frac{\delta}{\delta e^{C C^{\prime} j}}-i \hbar \frac{1}{4}\left[e_{A A^{\prime} i} \psi_{j}^{A}\right] e^{C C^{\prime} j} \frac{\delta}{\delta e^{C C^{\prime} i}}\right]
$$




$$
\begin{aligned}
& +\sqrt{2} \epsilon^{i j k} e_{A A^{\prime} i}{ }^{3(s)} \omega^{A}{ }_{B j} \psi^{B}{ }_{j} \\
& -\frac{i}{\sqrt{2}} \hbar n_{C A^{\prime}} \bar{\chi}^{C} \frac{\partial}{\partial \bar{\phi}} \\
& -i \sqrt{2} \hbar h e^{K / 2} P(\phi) n_{A^{\prime}}^{A} e_{A B^{\prime}} D^{C B^{\prime}}{ }_{j i} \frac{\partial}{\partial \psi_{j}^{C}} \\
& -i \sqrt{2} \hbar h e^{K / 2} D_{\phi} P n^{A}{ }_{A^{\prime}} \frac{\partial}{\partial \bar{\chi}^{A}} \\
& -\frac{i}{2 \sqrt{2}} h^{\frac{1}{2}} \hbar \phi n^{B B^{\prime}} n_{C B^{\prime}} \bar{\chi}^{C} n_{D A^{\prime}} \bar{\chi}^{D} \frac{\partial}{\partial \bar{\chi}^{B}} \\
& -\frac{i}{4 \sqrt{2}} \hbar h^{\frac{1}{2}} \phi \epsilon^{i j k} e^{B B_{j}^{\prime}} \psi_{k B} D^{C}{ }_{B^{\prime} l i} n_{D A^{\prime}} \bar{\chi}^{D} \frac{\partial}{\partial \psi_{l}^{C}} \\
& -\hbar \sqrt{2} h^{\frac{1}{2}} e^{B}{ }_{B^{\prime}}^{m} n^{C B^{\prime}} \psi_{m C} n_{D A^{\prime}} \bar{\chi}^{D} \frac{\partial}{\partial \bar{\chi}^{B}} \\
& -\frac{i}{\sqrt{2}} \hbar \epsilon^{i j k} e_{A A^{\prime} j} \psi_{i}^{A} n_{D B^{\prime}} \bar{\chi}^{D} e_{k}^{B B^{\prime}} \frac{\partial}{\partial \bar{\chi}^{B}} \\
& +\frac{1}{2 \sqrt{2}} \hbar h^{\frac{1}{2}} \psi_{i A}\left(e^{B}{ }_{A^{\prime}}{ }^{i} n^{A C^{\prime}}-e^{A C^{\prime} i} n_{A^{\prime}}^{B}\right) n_{D C^{\prime}} \bar{\chi}^{D} \frac{\partial}{\partial \bar{\chi}^{B}}, \\
& S_{A}=i \sqrt{2}\left[-\hbar^{2} \frac{1}{4 \sqrt{2}}\left[e_{A A^{\prime} i} h^{\frac{1}{2}} D_{k j}^{P A^{\prime}} \frac{\partial}{\partial \psi_{k}^{P}}\right] e^{C C^{\prime} i} \frac{\delta}{\delta e^{C C^{\prime} j}}\right. \\
& \left.-\hbar^{2} \frac{1}{4 \sqrt{2}}\left[e_{A A^{\prime} i} h^{\frac{1}{2}} D_{k j}^{P A^{\prime}} \frac{\partial}{\partial \psi_{k}^{P}}\right] e^{C C^{\prime} j} \frac{\delta}{\delta e^{C C^{\prime} i}}\right] \\
& -i \hbar \epsilon^{i j k} h^{\frac{1}{2}} e_{A A^{\prime} i}{ }^{3(s)} \bar{\omega}^{A^{\prime}{ }_{B^{\prime} j} D^{P B^{\prime}}{ }_{m k}} \frac{\partial}{\partial \psi_{m}^{P}} \\
& +i \hbar^{2} \frac{\partial}{\partial \bar{\chi}^{A}} \frac{\partial}{\partial \phi} \\
& -h^{\frac{1}{2}} e^{K / 2} P(\phi) n_{A}{ }^{A^{\prime}} e_{B A^{\prime}} \psi_{i}^{B} \\
& +i h^{\frac{1}{2}} e^{K / 2}\left(D_{\phi} P\right)^{*} n_{A}{ }^{A^{\prime}} n_{C A^{\prime}} \bar{\chi}^{C} \\
& -\frac{i}{2} h^{\frac{1}{2}} \hbar^{2} \bar{\phi} n^{B B^{\prime}} n_{D B^{\prime}} \bar{\chi}^{D} \frac{\partial}{\partial \bar{\chi}^{A}} \frac{\partial}{\partial \bar{\chi}^{B}} \\
& +\frac{i}{4} \hbar^{2} h^{\frac{1}{2}} \bar{\phi} \epsilon^{i j k} e_{j}^{B B_{j}^{\prime}} \psi_{i B} D_{B^{\prime} m k}^{C} \frac{\partial}{\partial \bar{\chi}^{A}} \frac{\partial}{\partial \psi_{m}^{C}}
\end{aligned}
$$




$$
\begin{aligned}
& +i \hbar^{2} h e_{B}^{B^{\prime} m} n^{B C^{\prime}} D^{C}{ }_{C^{\prime} k m} n_{D B^{\prime}} \bar{\chi}^{D} \frac{\partial}{\partial \bar{\chi}^{A}} \frac{\partial}{\partial \psi_{k}^{C}} \\
& +\frac{i}{2} \hbar^{2} \epsilon^{i j k} h^{\frac{1}{2}} e_{A A^{\prime} j} D_{m i}^{C A^{\prime} n_{D B^{\prime}}} \bar{\chi}^{D} e^{B B^{\prime}} \frac{\partial}{\partial \bar{\chi}^{B}} \frac{\partial}{\partial \psi_{m}^{C}} \\
& -\frac{i}{\sqrt{2}} \hbar^{2} h^{\frac{3}{2}}\left(e^{C A^{\prime} i} n_{A}^{B^{\prime}}-e_{A}^{B^{\prime} i} n^{C A^{\prime}}\right) n_{D B^{\prime}} \bar{\chi}^{D} D_{A^{\prime} m i}^{P} \frac{\partial}{\partial \bar{\chi}^{C}} \frac{\partial}{\partial \psi_{m}^{P}}
\end{aligned}
$$

where the terms containing no matter fields are consistent with ref. $[6,7,14,15,16]$. Notice that a constant analytical potential is similar to a cosmological constant term as in $[14,15,16]$.

The two above expressions will then be used together with (2.9), (2.12), to obtain the equations for the bosonic amplitudes coefficients on the wave function of the universe, $\Psi$. More precisely, we will employ the integrated form of the constraints in (2.4), i.e., $\mathcal{H} \equiv \int d^{3} x H$ obtained from the action re-written in a canonical form as $\mathcal{S}=\int d^{3} x(p \dot{q}-$ $H)$. We stress this point in order to deal correctly with terms as $\pi_{\phi} \bar{\chi}_{A}$ and hermitian conjugates and to obtain agreement with known expressions in dimensional-reduced models [9-12,18,21,25]. There is also another issue concerning the transformations (2.12) imposed by the matter fields $\chi, \bar{\chi}$ that should be pointed out. Similarly to ref.[15,16] we could try to analyse the case of FRW with complex scalar fields and fermionic partners using the Ansatz $\psi_{i}^{A}=e_{i}^{A A_{i}^{\prime}} \bar{\psi}_{A^{\prime}}[9-12,18,21,25]$. This implies (see eq. (3.16a)) that $\beta^{A}=\frac{3}{4} n^{A A^{\prime}} \bar{\psi}_{A^{\prime}} \sim \bar{\psi}^{A}$. However, the analysis in ref. [25] (see also [9-12,21]) from a one-dimensional reduced action point of view clearly indicates that we are required as well to redefine a new spin- $\frac{1}{2}$ component of the gravitino by $\bar{\psi}_{A} \rightarrow h^{\frac{1}{4}} \bar{\psi}_{A}$. Only with that we can properly obtain simple equations for the bosonic amplitudes. Consistently, a similar procedure has to be employed in this paper as it is easy to check. Hence, we have to consider a similar redefinition as (2.12) to the irreducible spin $\frac{1}{2}, \frac{3}{2}$ components of $\psi_{i}^{A}$. Such replacement is expected to give the correct supersymmetry constraints in the $k=1 \mathrm{FRW}$ model when it is quantized using the dimensional-reduction alternative approach via an adequate homogeneous Ansätze [9$12,21,25]$. Next, we address the construction of a Lorentz invariant Ansatz for $\Psi$, on which the supersymmetry constraints derived above will act.

The constraints $J^{A B} \Psi=0, \bar{J}^{A^{\prime} B^{\prime}} \Psi=0$ imply that $\Psi$ ought to be a Lorentz-invariant function. One takes then expressions in which all spinor indices have been contracted 
together. It is reasonable also to consider only wave functions $\Psi$ which are spatial scalars, where all spatial indices $i, j, \ldots$ have also been contracted together. To specify this, note the decomposition $[6,7,14,15,16]$ of $\psi_{B B^{\prime}}^{A}=e_{B B^{\prime}}^{i} \psi_{i}^{A}$ :

$$
\psi_{A B B^{\prime}}=-2 n_{B^{\prime}} \gamma_{A B C}+\frac{2}{3}\left(\beta_{A} n_{B B^{\prime}}+\beta_{B} n_{A B^{\prime}}\right)-2 \epsilon_{A B} n_{B^{\prime}} \beta_{C}
$$

where $\gamma_{A B C}=\gamma_{(A B C)}$ is totally symmetric and $\epsilon_{A B}$ is the alternating spinor.

As pointed out in the Introduction, we decide to construct our Lorentz invariant wave function (expressed in several fermionic sectors and corresponding bosonic amplitudes) using the framework represented in refs. [6-12,14-16,18,21,25]. We are aware of its limitations (they may be particularly severe in the case of $P(\phi) \neq 0$ ), as far as the middle sectors are concerned. In fact, we will be neglecting Lorentz invariants built with not only the spin $\frac{1}{2}$ and $\frac{3}{2}$ Lorentz irreducible mode components of the fermionic fields but also with the ones corresponding to the gravitational degrees of freedom. The new method proposed in $[23,24]$ do clarify some doubts and paradoxical situations in supersymmetric quantum cosmology, in particular constructing the correct middle fermionic sectors. However, the solutions in $[23,24]$ for the middle sectors are not entirely new, as explained in the Introduction. They were allready present in the "old" framework [8]. Thus, a simpler Lorentz invariant construction could still be of some utility, namely in order to accomodate the basic features of our model and to obtain new solutions. We hope these will correspond to states existing in ordinary minisuperspace quantum cosmology. Nevertheless, due care must be taken. In particular, if no states are allowed when $P(\phi) \neq 0$. The proper and complete treatment of our model would then have to follow ref.[23,24]. We may expect, however, that our results could be taken either as a complement or indication towards its implementation.

Our general Lorentz-invariant wave function is then taken to be a polynomial of eight degree in Grassmann variables

$$
\begin{gathered}
\Psi\left(a_{1}, a_{2}, a_{3}, \phi, \bar{\phi}\right)=A+B_{1} \beta_{A} \beta^{A}+B_{2} \bar{\chi}_{A} \bar{\chi}^{A} \\
+C_{1} \gamma_{A B C} \gamma^{A B C} \\
+D_{1} \beta_{A} \beta^{A} \gamma_{E B C} \gamma^{E B C}+D_{2} \bar{\chi}_{A} \bar{\chi}^{A} \gamma_{E B C} \gamma^{E B C}
\end{gathered}
$$




$$
\begin{gathered}
+E_{1}\left(\gamma_{A B C} \gamma^{A B C}\right)^{2} \\
F_{1}\left(\beta_{A} \beta^{A} \gamma_{E B C} \gamma^{E B C}\right)^{2}+F_{2} \bar{\chi}_{A} \bar{\chi}^{A}\left(\gamma_{E B C} \gamma^{E B C}\right)^{2} \\
+G_{1} \beta_{A} \beta^{A} \bar{\chi}_{B} \bar{\chi}^{B}+H_{1} \beta_{A} \beta^{A} \bar{\chi}_{B} \bar{\chi}^{B} \gamma_{E D C} \gamma^{E D C} \\
+I_{1} \beta_{A} \beta^{A} \bar{\chi}_{B} \bar{\chi}^{B}\left(\gamma_{E D C} \gamma^{E D C}\right)^{2} \\
+Z_{1} \bar{\chi}_{A} \beta^{A}+Z_{2} \bar{\chi}_{A} \beta^{A} \gamma_{E D C} \gamma^{E D C} \\
+Z_{3} \bar{\chi}_{A} \beta^{A}\left(\gamma_{E D C} \gamma^{E D C}\right)^{2}
\end{gathered}
$$

Note that the term $\left(\beta^{A} \gamma_{A B C}\right)^{2}=\beta^{A} \gamma_{A B C} \beta^{D} \gamma_{D}{ }^{B C}$ can be rewritten, using the anticommutation of the $\beta$ 's and $\gamma$ 's, as

$$
\text { const. } \beta^{E} \beta_{E} \epsilon^{A D} \gamma_{A B C} \gamma_{D}^{B C} \sim\left(\beta_{E} \beta^{E}\right)\left(\gamma_{A B C} \gamma^{A B C}\right) \text {. }
$$

Similarly, any quartic in $\gamma_{A B C}$ can be rewritten as a multiple of $\left(\gamma_{A B C} \gamma^{A B C}\right)^{2}$. Since there are only four independent components of $\gamma_{A B C}=\gamma_{(A B C)}$, only one independent quartic can be made from $\gamma_{A B C}$, and it is sufficient to check that $\left(\gamma_{A B C} \gamma^{A B C}\right)^{2}$ is nonzero. Now $\gamma_{A B C} \gamma^{A B C}=2 \gamma_{000} \gamma_{111}-6 \gamma_{100} \gamma_{011}$. Hence $\left(\gamma_{A B C} \gamma^{A B C}\right)^{2}$ includes a non-zero quartic term $\gamma_{000} \gamma_{100} \gamma_{110} \gamma_{111}$. The analytic potential imply that there is coupling between different fermionic levels.

Regarding the ordering chosen for the quantum mechanical operator form of the supersymmetry constraints $(3.10),(3.11)$ let us point the following. We decided to adopt the choice made in ref. $[17,18]$, namely that we order each term cubic in fermions in $\bar{S}_{A^{\prime}}$ (using anti-commutation) such that one fermionic derivative (momentum) is on the right and the fermionic variables are on the left. The ordering of the $S_{A}$ constraint is defined by taking the hermitian adjoint with respect to the natural inner product $[1,9-12,61]$; the terms in $S_{A}$ cubic in fermions have two derivatives (momenta) on the right and one fermionic variable (coordinate) on the left. We stress that the expressions (3.13), (3.14) still correspond to a full theory formulation (cf. ref. [6,7,14-18]). We could follow a different path, using a reduction through homogeneous Ansätze to get an action for a one one-dimensional time dependent model. In this context, one could choose an ordering as in [9-12], where all derivatives on the constraint corresponding to our $S_{A}$ is ordered with all derivatives on 
the left. In ref. [11] this ambiguity was taken into account and an expression for the supersymmetry constraints was defined, using again the hermitian adjoint with respect to the natural inner product $[1,61]$. Moreover, it related it to the previous one by putting extra linear tems in the fermionic coordinate and momenta. In [25] both the last two approaches were analysed for the case of a FRW model with supermatter and shown to be in agreement. We believe that our choice of ordering will produce equivalent results to those one could have obtained had we used a dimensional reduction from the start and possible different orderings. We will comment on this in the following and in section IV.

The action of the constraints operators $S_{A}, \bar{S}_{A^{\prime}}(3.10),(3.11)$ on $\Psi$ (3.13) leads to a system of coupled first order differential equations which the bosonic amplitude coefficients of $\Psi$ must satisfy. These coefficients are functions of $a_{1}, a_{2}, a_{3}, \phi, \bar{\phi}$. The equations are obtained after eliminating the $e_{i}^{A A^{\prime}}$ and $n^{A A^{\prime}}$ resulting in the $S_{A} \Psi=0, \bar{S}_{A^{\prime}} \Psi=0$ by contracting them with combinations of $e_{j}^{B B^{\prime}}$ and $n^{C C^{\prime}}$, following by integraton over $S^{3}$. These equations correspond essentially to expressions in front of terms such as $\bar{\chi}, \beta, \gamma, \beta^{A} \overline{\chi \chi}, \gamma^{A B C} \overline{\chi \chi}$, etc, after the fermionic derivatives in $S_{A}, \bar{S}_{A^{\prime}}$ have been performed on $S_{A} \Psi=0, \bar{S}_{A^{\prime}} \Psi=0$. As one can easily see, the number of obtained equations will be very large. Actually, its number will be $44 \times 3$, taking into account cyclic permutations on $a_{1}, a_{2}, a_{3}$ (see ref. [15,16]). Their full analysis is quite tedious and to write all the terms just would overburden the reader. Let us then instead describe the several steps involved in the calculations, showing as well some examples of the calculations involved in solving the $S_{A} \Psi=0, \bar{S}_{A^{\prime}} \Psi=0$ quantum constraints.

The supersymmetry constraints can be described as a combination of terms constituted by expressions in $e_{i}^{A A^{\prime}}, n^{A A^{\prime}}$ and $\phi, \bar{\phi}$, with fermionic variables. More precisely, the $\bar{S}_{A^{\prime}}$ constraint has fermionic terms of the following type: $\beta^{A}, \gamma^{A B C}, \bar{\chi}^{A}, \frac{\partial}{\partial \psi_{i}^{A}}, \frac{\partial}{\partial \bar{\chi}^{A}}$, $\bar{\chi} \bar{\chi} \frac{\partial}{\partial \bar{\chi}}, \psi \bar{\chi} \frac{\partial}{\partial \psi}, \psi \bar{\chi} \frac{\partial}{\partial \bar{\chi}}$. The $S_{A}$ constraint is of second order in fermionic derivatives and includes terms as: $\frac{\partial}{\partial \psi_{i}^{A}}, \frac{\partial}{\partial \bar{\chi}^{A}}, \beta^{A}, \gamma^{A B C}, \bar{\chi}^{A}, \bar{\chi} \frac{\partial}{\partial \bar{\chi}} \frac{\partial}{\partial \bar{\chi}}, \psi \frac{\partial}{\partial \bar{\chi}} \frac{\partial}{\partial \psi}, \bar{\chi} \frac{\partial}{\partial \bar{\chi}} \frac{\partial}{\partial \psi}$. In some of the expessions above we have deliberately not written some of the spatial or spinorial indexes as to allow for their possible combinations and contractions as one can see from the equations for $S_{A} \Psi=0, \bar{S}_{A^{\prime}} \Psi=0$. We will use the gravitino field written in terms of the $\beta \operatorname{spin} \frac{1}{2}$ and $\gamma$ spin $\frac{3}{2}$ modes, respectively. Moreover, we will use the following expressions: 


$$
\begin{gathered}
\frac{\partial\left(\beta_{A} \beta^{A}\right)}{\partial \psi^{B}{ }_{i}}=-n_{A}{ }^{\prime} e_{B B^{\prime}}{ }^{i} \beta^{A}, \\
\frac{\partial\left(\gamma_{A D C} \gamma^{A D C}\right)}{\partial \psi^{B}{ }_{i}}=-2 \gamma_{B D C} n^{C C^{\prime}} e^{D}{ }_{C^{\prime}}{ }^{i}, \\
\frac{\partial\left(\beta^{A} \beta_{A}\right)}{\partial \beta^{C}}=2 \beta^{C}, \\
\frac{\partial \beta_{A}}{\partial \psi^{B}{ }_{j}}=-\frac{1}{2} n_{A}{ }^{B^{\prime}} e_{B B^{\prime}}^{j}, \\
\frac{\partial \gamma^{A D C}}{\partial \psi^{B}{ }_{j}}=\frac{1}{3} n^{C C^{\prime}} e^{D}{ }_{C^{\prime}}{ }^{j} \epsilon_{B}{ }^{A}+\frac{1}{3} n^{A C^{\prime}} e^{C}{ }_{C^{\prime}}^{j} \epsilon_{B}{ }^{D}+\frac{1}{3} n^{D C^{\prime}} e^{A}{ }_{C^{\prime}}^{j} \epsilon_{B}{ }^{C} .
\end{gathered}
$$

We also write out $\beta^{A}$ and $\gamma_{B D C}$ in terms of $e^{E E^{\prime}}$ and $\psi^{E}{ }_{j}$ as

$$
\begin{gathered}
\beta_{A}=-\frac{1}{2} n_{A}^{A^{\prime} i} e_{B A^{\prime}}^{i} \psi_{i}^{B} \\
\gamma_{A B C}=\frac{1}{3} n_{C}^{C^{\prime}} e_{B C^{\prime}}^{i} \psi_{A i}+\frac{1}{3} n_{A}^{C^{\prime}} e_{C C^{\prime}}^{i} \psi_{B i}+\frac{1}{3} n_{B}^{C^{\prime}} e_{A C^{\prime}}^{i} \psi_{C i}
\end{gathered}
$$

from which (3.15a), (3.15b) become

$$
\begin{gathered}
\frac{\partial\left(\beta_{A} \beta^{A}\right)}{\partial \psi_{i}^{B}}=\frac{1}{4} e_{B B^{\prime}}^{i} e_{C}^{B^{\prime} j} \psi_{j}^{C}, \\
\frac{\partial\left(\gamma_{A D C} \gamma^{A D C}\right)}{\partial \psi_{i}^{B}}=-\frac{1}{3} e_{D A^{\prime}}^{j} e^{D A^{\prime} i} \psi_{B j} \\
-\frac{2}{3} n_{B}^{A^{\prime}} n^{C C^{\prime}} e_{C A^{\prime}}^{j} e^{D}{ }_{C^{\prime}}^{i} \psi_{D j}+\frac{1}{3} e_{B A^{\prime}}^{j} e^{C A^{\prime} i} \psi_{C j} .
\end{gathered}
$$

Since the wave function is of even order in fermionic variables, the equations $S_{A} \Psi=$ $0, \bar{S}_{A^{\prime}} \Psi=0$ will be of odd order in fermionic variables, up to seventh order. Notice that some of the fermionic terms in (3.10), (3.11) applied to $\Psi$ increase the fermionic order by a factor of one (e.g, $\bar{\chi})$ while others as $\bar{\chi} \frac{\partial}{\partial \bar{\chi}} \frac{\partial}{\partial \psi}$ decrease it by the same amount.

The two following tables illustrate in a simple way how the quantum supersymmetry constraints $(3.10),(3.11)$ operate on $\Psi(3.13)$ and which types of fermionic terms can be 
obtained. The first table correspond to $S_{A} \Psi$ and the second to $\bar{S}_{A^{\prime}} \Psi$. The first row is constituted by the fermionic operators present in the supersymmetry constraints while in the first column we have the several terms which are consequently obtained from $S_{A} \Psi=$ $0, \bar{S}_{A^{\prime}} \Psi=0$. In the intersection slots we have the possible different bosonic coefficients of the wave function $\Psi$, representing the specific way a fermionic operator acts on $\Psi$. For example, we see that the operator $\bar{\chi}$ in $\bar{S}_{A^{\prime}}$ produces fermionic terms such as $\beta \overline{\chi \chi}$, $\beta \overline{\chi \chi} \gamma \gamma, \beta \overline{\chi \chi}(\gamma \gamma)^{2}$ to which correspond bosonic functions $Z_{1}, Z_{2}, Z_{3}$, respectively. In such a way we can infer which type of wave function coefficients are present in each equation (say, the one corresponding to $\bar{\chi} \beta \beta(\gamma \gamma)^{2}$ ) and which fermionic operators have produced those terms. The brackets in $(\gamma \gamma)$ stand for $\gamma^{A B C} \gamma_{A B C}$ and the same notation will be used for the other fermionic variables throughout the paper. A slash over a particular bosonic amplitude means that after all the calculations have been made to simplify the corresponding equations, the expression associated with that particular coefficient is zero. An example is when those expressions contain something like $\gamma^{A B C} \epsilon_{B C}$ which is zero due to the symmetry of $\gamma^{A B C}=\gamma^{(A B C)}$ and the antisymmetry of $\epsilon_{A B}=\epsilon_{[A B]}$. Hence, they do not contribute to the set of equations we will discuss in the following two subsections. A "•" means that no bosonic amplitudes in $\Psi$ can match the particular fermionic operator and the term in $S_{A} \Psi=0, \bar{S}_{A^{\prime}} \Psi=0$, respectively. As a last comment, the third and fourth columns in both tables corespond to the action of fermionic operators which appear in terms involving $P(\phi), D_{\phi} P(\phi)$ respectively, or their hermitian conjugates. 


\begin{tabular}{|c|c|c|c|c|c|c|c|}
\hline$\searrow$ & $\frac{\partial}{\partial \psi}$ & $\frac{\partial}{\partial \bar{\chi}}$ & $\psi$ & $\bar{\chi}$ & $\bar{\chi} \frac{\partial}{\partial \bar{\chi}} \frac{\partial}{\partial \bar{\chi}}$ & $\psi \frac{\partial}{\partial \bar{\chi}} \frac{\partial}{\partial \psi}$ & $\bar{\chi} \frac{\partial}{\partial \bar{\chi}} \frac{\partial}{\partial \psi}$ \\
\hline$\beta$ & $B_{1}$ & $Z_{1}$ & $A$ & $\bullet$ & $\bullet$ & $Z_{1}$ & $\bullet$ \\
\hline$\gamma$ & $C_{1}$ & $\bullet$ & A & $\bullet$ & $\bullet$ & $Z_{1}$ & $\bullet$ \\
\hline $\bar{\chi}$ & $Z_{1}$ & $B_{2}$ & $\bullet$ & $A$ & $B_{2}$ & $\bullet$ & $Z_{1}$ \\
\hline$\beta \beta \bar{\chi}$ & $\bullet$ & $G_{1}$ & $Z_{1}$ & $B_{1}$ & $\phi_{1}$ & $\phi_{1}$ & $\bullet$ \\
\hline$\beta \beta \gamma$ & $D_{1}$ & $\bullet$ & $\not B_{1}$ & $\bullet$ & $\bullet$ & $Z_{2}$ & $\bullet$ \\
\hline$\beta \overline{\chi \chi}$ & $G_{1}$ & $\bullet$ & $B_{2}$ & $Z_{1}$ & $\bullet$ & $\bullet$ & $\phi_{1}$ \\
\hline$\beta \bar{\chi} \gamma$ & $Z_{2}$ & $\bullet$ & $\bullet$ & $\bullet$ & $\bullet$ & $D_{2}, G_{1}$ & $Z_{2}$ \\
\hline$\beta \beta \gamma$ & $D_{1}$ & $Z_{2}$ & $C_{1}$ & $\bullet$ & $\bullet$ & $Z_{2}$ & $\bullet$ \\
\hline$\overline{\chi \chi} \gamma$ & $D_{2}$ & $\bullet$ & $\not B_{2}$ & $\bullet$ & $\bullet$ & $\bullet$ & $\not D_{2}$ \\
\hline $\bar{\chi} \gamma \gamma$ & $Z_{2}$ & $D_{2}$ & $\bullet$ & $C_{1}$ & $D_{2}$ & $D_{2}$ & $\bullet$ \\
\hline$\gamma \gamma \gamma$ & $E_{1}$ & $\bullet$ & $\phi_{1}$ & $\bullet$ & $\bullet$ & $Z_{2}$ & $\bullet$ \\
\hline$\beta(\gamma \gamma)^{2}$ & $F_{1}$ & $Z_{3}$ & $E_{1}$ & $\bullet$ & - & $Z_{3}$ & - \\
\hline$\gamma \beta \beta \gamma \gamma$ & $F_{1}$ & $\bullet$ & $\not D_{1}$ & $\bullet$ & $\bullet$ & $Z_{3}$ & $\bullet$ \\
\hline$\gamma \overline{\chi \chi} \gamma \gamma$ & $F_{2}$ & $\bullet$ & $\not D_{2}$ & $\bullet$ & $\bullet$ & $\bullet$ & $\mathrm{H}_{2}$ \\
\hline$\beta \overline{\chi \chi} \gamma \gamma$ & $H_{1}$ & $\bullet$ & $D_{2}$ & $Z_{2}$ & $\bullet$ & $\bullet$ & $H_{1}$ \\
\hline$\gamma \beta \beta \overline{\chi \chi}$ & $H_{1}$ & $\bullet$ & $Q_{1}$ & $\bullet$ & $\bullet$ & $\bullet$ & $\not H_{1}$ \\
\hline $\bar{\chi}(\gamma \gamma)^{2}$ & $Z_{3}$ & $F_{2}$ & $\bullet$ & $E_{1}$ & $F_{2}$ & $F_{2}$ & $Z_{3}$ \\
\hline $\bar{\chi} \beta \gamma \gamma \gamma$ & $Z_{3}$ & $\bullet$ & $\bullet$ & $\bullet$ & $\bullet$ & $F_{2}, H_{1}$ & $Z_{3}$ \\
\hline $\bar{\chi} \beta \beta \gamma \gamma$ & $\bullet$ & $H_{1}$ & $Z_{2}$ & $\bullet$ & $\not H_{1}$ & $H_{1}$ & • \\
\hline$\beta \overline{\chi \chi}(\gamma \gamma)^{2}$ & $I_{1}$ & $\bullet$ & $F_{2}$ & $Z_{3}$ & $\bullet$ & $\bullet$ & $\mathbf{I}_{1}$ \\
\hline$\gamma \beta \beta \overline{\chi \chi} \gamma \gamma$ & $I_{1}$ & $\bullet$ & $H_{1}$ & $\bullet$ & $\bullet$ & $\bullet$ & $\mathbf{I}_{1}$ \\
\hline $\bar{\chi} \beta \beta(\gamma \gamma)^{2}$ & - & $I_{1}$ & $Z_{3}$ & $F_{1}$ & $\mathbf{I}_{1}$ & $\mathbf{I}_{1}$ & $\bullet$ \\
\hline
\end{tabular}

Table 1: Action of $S_{A}$ (3.11) on $\Psi$ (3.13) 


\begin{tabular}{|c|c|c|c|c|c|c|c|}
\hline$\searrow$ & $\psi$ & $\bar{\chi}$ & $\frac{\partial}{\partial \psi}$ & $\frac{\partial}{\partial \bar{\chi}}$ & $\overline{\chi \chi} \frac{\partial}{\partial \bar{\chi}}$ & $\psi \bar{\chi} \frac{\partial}{\partial \psi}$ & $\psi \bar{\chi} \frac{\partial}{\partial \bar{\chi}}$ \\
\hline$\beta$ & $A$ & $\bullet$ & $B_{1}$ & $Z_{1}$ & $\bullet$ & $\bullet$ & $\bullet$ \\
\hline$\gamma$ & $A$ & $\bullet$ & $\phi_{1}$ & $\bullet$ & $\bullet$ & $\bullet$ & $\bullet$ \\
\hline $\bar{\chi}$ & $\bullet$ & $A$ & $Z_{1}$ & $B_{2}$ & $\bullet$ & $\bullet$ & $\bullet$ \\
\hline$\beta \beta \bar{\chi}$ & $Z_{1}$ & $B_{1}$ & $\bullet$ & $G_{1}$ & $\bullet$ & $B_{1}$ & $Z_{1}$ \\
\hline$\beta \beta \gamma$ & $B_{1}$ & $\bullet$ & $D_{1}$ & $\bullet$ & $\bullet$ & $\bullet$ & $\bullet$ \\
\hline$\beta \overline{\chi \chi}$ & $B_{2}$ & $Z_{1}$ & $G_{1}$ & $\bullet$ & $Z_{1}$ & $Z_{1}$ & $B_{2}$ \\
\hline$\beta \bar{\chi} \gamma$ & $Z_{1}$ & $\bullet$ & $\bullet$ & $\bullet$ & $\bullet$ & $C_{1} B_{1}$ & $Z_{1}$ \\
\hline$\beta \gamma \gamma$ & $C_{1}$ & $\bullet$ & $D_{1}$ & $Z_{2}$ & $\bullet$ & $\bullet$ & $\bullet$ \\
\hline$\overline{\chi \chi} \gamma$ & $B_{2}$ & $\bullet$ & $D_{2}$ & $\bullet$ & $\bullet$ & $Z_{1}$ & $B_{2}$ \\
\hline $\bar{\chi} \gamma \gamma$ & $\bullet$ & $C_{1}$ & $Z_{2}$ & $D_{2}$ & $\bullet$ & $C_{1}$ & $\bullet$ \\
\hline$\gamma \gamma \gamma$ & $C_{1}$ & $\bullet$ & $E_{1}$ & $\bullet$ & $\bullet$ & $\bullet$ & $\bullet$ \\
\hline$\beta(\gamma \gamma)^{2}$ & $E_{1}$ & $\bullet$ & $F_{1}$ & $Z_{3}$ & $\bullet$ & $\bullet$ & $\bullet$ \\
\hline$\gamma \beta \beta \gamma \gamma$ & $D_{1}$ & $\bullet$ & $F_{1}$ & $\bullet$ & $\bullet$ & $\bullet$ & $\bullet$ \\
\hline$\gamma \overline{\chi \chi} \gamma \gamma$ & $D_{2}$ & $\bullet$ & $F_{2}$ & $\bullet$ & $\bullet$ & $Z_{2}$ & $D_{2}$ \\
\hline$\beta \overline{\chi \chi} \gamma \gamma$ & $D_{2}$ & $Z_{2}$ & $H_{1}$ & $\bullet$ & $Z_{2}$ & $Z_{2}$ & $D_{2}$ \\
\hline$\gamma \beta \beta \overline{\chi \chi}$ & $G_{1}$ & $\bullet$ & $H_{1}$ & $\bullet$ & $\bullet$ & $Z_{2}$ & $H_{1}$ \\
\hline $\bar{\chi}(\gamma \gamma)^{2}$ & $\bullet$ & $E_{1}$ & $Z_{3}$ & $F_{2}$ & $\bullet$ & $E_{1}$ & $\bullet$ \\
\hline $\bar{\chi} \beta \gamma \gamma \gamma$ & $Z_{2}$ & $\bullet$ & $\bullet$ & $\bullet$ & $\bullet$ & $D_{1}, E_{1}$ & $Z_{2}$ \\
\hline $\bar{\chi} \beta \beta \gamma \gamma$ & $Z_{2}$ & $D_{1}$ & $\bullet$ & $H_{1}$ & $\bullet$ & $D_{1}$ & $Z_{2}$ \\
\hline$\beta \overline{\chi \chi}(\gamma \gamma)^{2}$ & $F_{2}$ & $Z_{3}$ & $I_{1}$ & $\bullet$ & $Z_{3}$ & $Z_{3}$ & $F_{2}$ \\
\hline$\gamma \beta \beta \overline{\chi \chi} \gamma \gamma$ & $H_{1}$ & $\bullet$ & $\mathbf{I}_{1}$ & $\bullet$ & $\bullet$ & $\bullet$ & $H_{1}$ \\
\hline $\bar{\chi} \beta \beta(\gamma \gamma)^{2}$ & $Z_{3}$ & $F_{1}$ & $\bullet$ & $I_{1}$ & $\bullet$ & $F_{1}$ & $Z_{3}$ \\
\hline
\end{tabular}

Table 2: Action of $\bar{S}_{A^{\prime}}(3.10)$ on $\Psi(3.13)$

It is worthwhile to stress the following important property, which holds regardless we put $P(\phi)=0$ or not. Using the symmetry properties of $e_{i}^{A A^{\prime}}, n_{A A^{\prime}}, \gamma^{A B C}, \epsilon_{A B}$ we can check that all equations which correspond to the terms $\gamma, \gamma \beta \beta, \overline{\chi \chi} \gamma, \gamma \gamma \gamma, \gamma \beta \beta \gamma \gamma, \gamma \overline{\chi \chi} \gamma \gamma$, $\gamma \beta \beta \overline{\chi \chi}, \gamma \beta \beta \overline{\chi \chi} \gamma \gamma$ in $S_{A} \Psi=0, \bar{S}_{A^{\prime}} \Psi=0$ will give a similar expression for the coefficients 
$A, B_{1}, B_{2}, C_{1}, D_{1}, D_{2}, E_{1}, F_{1}, F_{2}, G_{1}, H_{1}, I_{1}$. Namely,

$$
P\left(a_{1}, a_{2}, a_{3} ; \phi, \bar{\phi}\right) e^{ \pm\left(a_{1}^{2}+a_{2}^{2}+a_{3}^{2}\right)}
$$

(cf.ref. $[7,8,23,24]$ and see below). The same does not apply to the $Z_{1}, Z_{2}, Z_{3}$ coefficients as the $\beta \bar{\chi} \gamma$ and $\beta \bar{\chi} \gamma(\gamma \gamma)$ terms from both the supersymmetry constraints just mix them with other coefficients in $\Psi$.

In the following we will analyse two cases separately: when the analytic potential $P(\Phi)$ is arbitrarly and when is identically set to zero. We will begin by the former.

\section{Case $\mathcal{A} \cdot P(\phi) \neq 0$}

From the equations corresponding to $\gamma_{D E F}(\beta \beta)$ and $\gamma_{F G H}(\gamma \gamma)$ in $\bar{S}_{A^{\prime}} \Psi=0$ we get

$$
\begin{gathered}
2 \epsilon^{i j k} e_{A A^{\prime} i} \omega_{B j}^{A} n_{B^{\prime}} e^{C B^{\prime}{ }_{k} B_{1}-\hbar n^{D}{ }_{B^{\prime}} e^{C B^{\prime}}}{ }_{i} \frac{\delta B_{1}}{\delta e^{B A^{\prime}}}{ }_{i} \\
+(B C D \rightarrow C D B)+(B C D \rightarrow D B C)=0 \\
2 \epsilon^{i j k} e_{A A^{\prime} i} \omega^{A}{ }_{B j} n^{D}{ }_{B^{\prime}} e^{C B^{\prime}}{ }_{k} C_{1}-\hbar n^{D}{ }_{B^{\prime}} e^{C B^{\prime}}{ }_{i} \frac{\delta C_{1}}{\delta e^{B A^{\prime}}}{ }_{i} \\
+(B C D \rightarrow C D B)+(B C D \rightarrow D B C)=0 .
\end{gathered}
$$

Contracting Eq. (3.18), (3.19) with $e^{B A^{\prime} \ell} n_{C C^{\prime}} e_{D}{ }^{C^{\prime} N}$, multiplying by, say,

$$
\delta h_{\ell n}=\frac{\partial h_{\ell n}}{\partial a_{1}}=2 a_{1} E_{i}^{1} E_{j}^{1}
$$

and integrating over $S^{3}$ gives

$$
\begin{gathered}
3 \hbar a_{1} \frac{\partial B_{1}}{\partial a_{1}}-\hbar\left(a_{1} \frac{\partial B_{1}}{\partial a_{1}}+a_{2} \frac{\partial B_{1}}{\partial a_{2}}+a_{3} \frac{\partial B_{1}}{\partial a_{3}}\right) \\
-16 \pi^{2} a_{1} a_{2} a_{3}\left(\frac{a_{3}}{a_{1} a_{2}}+\frac{a_{2}}{a_{3} a_{1}}-2 \frac{a_{1}}{a_{2} a_{3}}\right) B_{1}=0,
\end{gathered}
$$




$$
\begin{aligned}
& 3 \hbar a_{1} \frac{\partial C_{1}}{\partial a_{1}}-\hbar \kappa^{2}\left(a_{1} \frac{\partial C_{1}}{\partial a_{1}}+a_{2} \frac{\partial C_{1}}{\partial a_{2}}+a_{3} \frac{\partial C_{1}}{\partial a_{3}}\right) \\
& -16 \pi^{2} a_{1} a_{2} a_{3}\left(\frac{a_{3}}{a_{1} a_{2}}+\frac{a_{2}}{a_{3} a_{1}}-2 \frac{a_{1}}{a_{2} a_{3}}\right) C_{1}=0,
\end{aligned}
$$

and the corresponding equations given by permuting $a_{1} a_{2} a_{3}$ cyclically.

Following the steps in ref. $[14,15,16]$, we consider now eq. (3.20), (3.21) and cyclic permutations. These leads to

$$
\begin{aligned}
& \hbar\left(a_{1} \frac{\partial B_{1}}{\partial a_{1}}-a_{2} \frac{\partial B_{1}}{\partial a_{2}}\right)=16 \pi^{2}\left(a_{2}^{2}-a_{1}^{2}\right) B_{1} \\
& \hbar\left(a_{1} \frac{\partial C_{1}}{\partial a_{1}}-a_{2} \frac{\partial C_{1}}{\partial a_{2}}\right)=16 \pi^{2}\left(a_{2}^{2}-a_{1}^{2}\right) C_{1}
\end{aligned}
$$

and cyclic permutations. Integrating them along a characteristic $a_{1} a_{2}=$ const., $a_{3}=$ const., using the parametric description $a_{1}=u_{1} e^{\tau}, a_{2}=u_{2} e^{-\tau}$, we get

$$
B_{1}=f\left(a_{1} a_{2}, a_{3} ; \phi, \bar{\phi}\right) e^{-\frac{8 \pi^{2}}{\hbar}\left(a_{1}^{2}+a_{2}^{2}\right)}
$$

and similarly to $C_{1}$. From their cyclic permutations and from the invariance under this cyclic permutation over $a_{1}, a_{2}, a_{3}$ we subsquently obtain

$$
\begin{aligned}
& B_{1}=f\left(a_{1} a_{2} a_{3} ; \phi, \bar{\phi}\right) e^{-\frac{8 \pi^{2}}{\hbar}\left(a_{1}^{2}+a_{2}^{2}+a_{3}^{2}\right)}, \\
& C_{1}=g\left(a_{1} a_{2} a_{3} ; \phi, \bar{\phi}\right) e^{-\frac{8 \pi^{2}}{\hbar}}\left(a_{1}^{2}+a_{2}^{2}+a_{3}^{2}\right)
\end{aligned}
$$

The same type of expressions follow for the remaining bosonic coefficients with the exception of $Z_{1}, Z_{2}, Z_{3}$.

Let us then proceed considering the equations obtained from $S_{A} \Psi=0$ and first order in fermions, with terms linear in $\beta$ and $\gamma$. These equations (see also next subsection), after contraction with expressions in $e_{i}^{A A^{\prime}}, n_{A A^{\prime}}$ and integrating over $S^{3}$ and using (3.15)-(3.17), can be combined into

$$
\begin{aligned}
& \frac{1}{16} \hbar^{2}\left(a_{1} \frac{\partial B_{1}}{\partial a_{1}}+a_{2} \frac{\partial B_{1}}{\partial a_{2}}+a_{3} \frac{\partial B_{1}}{\partial a_{3}}\right) \\
- & \frac{1}{3} \hbar a_{1}\left[3 \frac{\partial C_{1}}{\partial a_{1}}-a_{1}^{-1}\left(a_{1} \frac{\partial C_{1}}{\partial a_{1}}+a_{2} \frac{\partial C_{1}}{\partial a_{2}}+a_{3} \frac{\partial C_{1}}{\partial a_{3}}\right)\right]
\end{aligned}
$$




$$
\begin{aligned}
& -16 \pi^{2} e^{K / 2} P(\phi) a_{1} a_{2} a_{3} A-\pi^{2} \hbar a_{1} a_{2} a_{3}\left(\frac{a_{1}}{a_{2} a_{3}}+\frac{a_{2}}{a_{3} a_{1}}+\frac{a_{3}}{a_{1} a_{2}}\right) B_{1} \\
& +\frac{1}{3}\left(16 \pi^{2}\right) \hbar a_{1} a_{2} a_{3}\left(\frac{2 a_{1}}{a_{2} a_{3}}-\frac{a_{2}}{a_{3} a_{1}}-\frac{a_{3}}{a_{1} a_{2}}\right) C_{1} \\
& +8 \pi^{2} \bar{\phi} \hbar^{2} Z_{1}-2 i \hbar^{2} \frac{\partial Z_{1}}{\partial \phi}=0
\end{aligned}
$$

and two more equations given by cyclic permutation of $a_{1} a_{2} a_{3}$. Let us point out that the $\beta$ equation correspond to the trace of (3.27) while the $\gamma$ one represents its trace free part. Eq. (3.27) differs from the one in ref. [15,16] in its last two terms. From eq. (3.25), (3.26) and (3.27) with cyclic permutations we get

$$
\begin{aligned}
& 16 \pi^{2} e^{K / 2} P(\phi) A=-2 \pi^{2} \hbar\left(a_{1} a_{2} a_{3}\right)^{-1}\left(a_{1}^{2}+a_{2}^{2}+a_{3}^{2}\right) e^{\left[-\frac{8 \pi^{2}}{\hbar}\left(a_{1}^{2}+a_{2}^{2}+a_{3}^{2}\right)\right]} f \\
& +\frac{3}{16} \hbar^{2} \kappa^{2} e^{\left[-\frac{8 \pi^{2}}{\hbar}\left(a_{1}^{2}+a_{2}^{2}+a_{3}^{2}\right)\right]} f^{\prime} \\
& +\frac{2}{3}\left(16 \pi^{2}\right) \hbar\left(a_{1} a_{2} a_{3}\right)^{-1}\left(2 a_{1}^{2}-a_{2}^{2}-a_{3}^{2}\right) e^{\left[-\frac{8 \pi^{2}}{\hbar}\left(a_{1}^{2}+a_{2}^{2}+a_{3}^{2}\right)\right]} g \\
& +8 \pi^{2} \hbar^{2}\left(a_{1} a_{2} a_{3}\right)^{-1} \phi Z_{1}-2 i \hbar^{2}\left(a_{1} a_{2} a_{3}\right)^{-1} \frac{\partial Z_{1}}{\partial \phi}
\end{aligned}
$$

and cyclically. We assume as well as in $[14,15,16]$ that the coefficients in the the wave function are invariant under permutations of $a_{1}, a_{2}, a_{3}$. We then get $g=0 \Rightarrow C_{1}=0$ as the only possible solution.

Now, eq. (3.28) and its cyclic permutations with $C_{1}=0$ must be solved consistently with the equation obtained from the linear terms in $\beta$ and $\gamma$ from $\bar{S}_{A^{\prime}} \Psi=0$. We follow the same procedure as above, writting $\beta$ and $\gamma$ in terms of $\psi_{A}{ }^{i}$, adding them and geting

$$
\hbar a_{1} \frac{\partial A}{\partial a_{1}}+16 \pi^{2} a_{1}^{2} A+6 \pi^{2} \hbar e^{K / 2} P(\phi) a_{1} a_{2} a_{3} B_{1}-\sqrt{2} 16 \pi^{2} \hbar a_{1} a_{2} a_{3} e^{K / 2}\left(D_{\phi} P(\phi)\right) Z_{1}=0
$$

and two others given by cyclic permutation of $a_{1} a_{2} a_{3}$. Relatively to ref. $[14,15,16]$, the main difference is in the term in $Z_{1}$. Eliminating $A$, we get

$$
\begin{aligned}
& \frac{3 \hbar^{3}}{16\left(16 \pi^{2} e^{K / 2} P(\phi)\right)} f^{\prime \prime}-\frac{\hbar^{2}}{8 e^{K / 2} P(\phi)} \frac{\left(a_{1}^{2}+a_{2}^{2}+a_{3}^{2}\right)}{a_{1} a_{2} a_{3}} f^{\prime} \\
& +6 \pi^{2} \hbar e^{K / 2} P(\phi) f-\frac{\hbar^{2}}{4 e^{K / 2} P(\phi)} \frac{1}{a_{2}^{2} a_{3}^{2}} f+\frac{\hbar^{2}}{8 e^{K / 2} P(\phi)} \frac{\left(a_{1}^{2}+a_{2}^{2}+a_{3}^{2}\right)}{\left(a_{1} a_{2} a_{3}\right)^{2}} f
\end{aligned}
$$




$$
\begin{aligned}
& -\frac{\hbar^{3}}{16 \pi^{2}} \frac{1}{e^{K / 2} P(\phi)} \frac{1}{\left(a_{1} a_{2} a_{3}\right)^{2}} \bar{\phi} e^{\left[\frac{8 \pi^{2}}{\hbar}\left(a_{1}^{2} a_{2}^{2}+a_{3}^{2}\right)\right]} Z_{1} \\
& \frac{\hbar^{2}}{2 e^{K / 2} P(\phi)} \bar{\phi} e^{\left[\frac{8 \pi^{2}}{\hbar}\left(a_{1}^{2} a_{2}^{2}+a_{3}^{2}\right)\right]} \frac{Z_{1}}{a_{2}^{2} a_{3}^{2}} \\
& +\frac{2 i \hbar^{3}}{16 \pi^{2}} \frac{1}{e^{K / 2} P(\phi)} \frac{1}{\left(a_{1} a_{2} a_{3}\right)^{2}} e^{\left[\frac{8 \pi^{2}}{\hbar}\left(a_{1}^{2}+a_{2}^{2}+a_{3}^{2}\right)\right]} \frac{\partial Z_{1}}{\partial \phi} \\
& -\frac{2 i \hbar^{2}}{e^{K / 2} P(\phi)} e^{\left[\frac{8 \pi^{2}}{\hbar}\left(a_{1}^{2} a_{2}^{2}+a_{3}^{2}\right)\right]} \frac{1}{a_{2}^{2} a_{3}^{2}} \frac{\partial Z_{1}}{\partial \phi} \\
& -\sqrt{2} 16 \pi^{2} i \hbar e^{K / 2} D_{\phi} P(\phi) e^{\left[\frac{8 \pi^{2}}{\hbar}\left(a_{1}^{2} a_{2}^{2}+a_{3}^{2}\right)\right]} Z_{1}=0,
\end{aligned}
$$

and cyclic permutations. Since $f=f\left(a_{1} a_{2} a_{3} ; \phi, \bar{\phi}\right)$ is invariant under permutations of $a_{1}, a_{2}, a_{3}$, the terms in $\left(a_{2} a_{3}\right)^{-2} f$ term and its permutations imply now, differently from $[14,15,16]$, a relation between $Z_{1}$ and $B_{1}$ :

$$
f\left(a_{1} a_{2} a_{3} ; \phi, \bar{\phi}\right) e^{\left[\frac{8 \pi^{2}}{\hbar}\left(a_{1}^{2} a_{2}^{2}+a_{3}^{2}\right)\right]}+2 \bar{\phi} Z_{1}-8 i \frac{\partial Z_{1}}{\partial \phi}=0 .
$$

For the particular case of $B_{1}=0$, i.e., $(f=0)$, it follows in the end of the day that from the remaining equations the only possible solution is $\Psi=0$. Considering the equations from the linear term in $\beta$ and $\gamma$ from $\bar{S}_{A^{\prime}} \Psi=0$, the only term different from ref. $[14,15,16]$ is the one in $Z_{1}$. But it happens that the solutions of the equation in $\gamma$ are the same as in ref. [7] (cf. eq. (3.18)-(3.26)). So, substituting these solution back in the equation (3.29) - see also table 2 for the $\beta$ equation - all terms safe the last will contribute to give zero as they are allready present in the corresponding equation for the Bianchi-IX model with $\Lambda=0$ [7]. Hence, we are left out with $Z_{1}=0$. As a consequence, the equation corresponding to the term in $S_{A} \Psi=0$ linear in $\beta$ gives then $A=0$. Using all these results in the equation corresponding to the tems linear in $\bar{\chi}$ from $\bar{S}_{A^{\prime}} \Psi=0$ gives $B_{2}=0$. Then the $\beta \beta \bar{\chi}$ term from $\bar{S}_{A^{\prime}} \Psi=0$ implies $G_{1}=0$. Consequently the $\gamma \beta \beta \overline{\chi \chi}$ term from the $\bar{S}_{A^{\prime}}$ constraint leads to $Z_{2}=0$. The $\beta \bar{\chi} \gamma$ term from $S_{A}$ gives that $D_{2}=0$. Again, collecting these results in the $\beta \gamma \gamma$ term from $\bar{S}_{A^{\prime}} \Psi=0$ we obtain $D_{1}=0$. The $\beta \overline{\chi \chi} \gamma \gamma$ term from $\bar{S}_{A^{\prime}} \Psi=0$ leads to $H_{1}=0$ and the $\beta \bar{\chi} \gamma \gamma \gamma$ term to $E_{1}=0$. Finally, we have to address the coefficients $F_{1}, F_{2}, I_{1}, Z_{3}$ of $\Psi$. Their analysis turn out to be similar to the one of $A, B_{1}, B_{2}, Z_{1}$ but without the $C_{1}$ coefficient. Using the equations corresponding 
to $\beta \overline{\chi \chi}(\gamma \gamma)^{2}$ from $\bar{S}_{A^{\prime}} \Psi=0, \gamma \overline{\chi \chi} \gamma \gamma, \gamma \beta \beta \gamma \gamma$ and $\beta \overline{\chi \chi}(\gamma \gamma)^{2}$ terms in $S_{A} \Psi=0$ we get that $F_{2}=0$. Then $\bar{\chi}(\gamma \gamma)^{2}$ in $\bar{S}_{A^{\prime}} \Psi=0, \beta(\gamma \gamma)^{2}$ in $\bar{S}_{A^{\prime}} \Psi=0$ and $\beta \bar{\chi} \beta(\gamma \gamma)^{2}$ in $\bar{S}_{A^{\prime}} \Psi=0$ induce that $Z_{3}=0, F_{2}=0$ and $I_{1}=0$, respectively. However, these results are just a consequence of a rather particular case. We should address then a more general situation.

For an arbitrarly $f$, eq. (3.31) allows to write an expression for $Z_{1}$ in terms of functions of $\phi, \bar{\phi}$ and $a_{1}, a_{2}, a_{3}$. If we use that expression in other equations, we get other formulas for other bosonic coefficients. For example, from the equation in $\beta$ from $\bar{S}_{A^{\prime}} \Psi=0$ we may use $B_{1}, Z_{1}$ as above to get an expression for $A$. One complements these steps with the equation linear in $\bar{\chi}$ from $\bar{S}_{A^{\prime}} \Psi=0$ and study $B_{2}$. Following this procedure, we would get the general solution of this extremely complicated set of differential equations. Although apparently possible, we could not establish a definite result in the end due to the complexity of the equations involved. As in [10,12], no easy way is apparent of obtaining an analitycal solution to this set of equations. Moreover, the exponential terms $e^{K / 2}$ leads to some difficulties.

We could also speculate by saying that non trivial physical states could be found for a generic $P(\phi)$. In fact, the presence of the anisotropic gravitational degrees of freedom and the spin $\frac{3}{2}$ modes of the gravitino together with the matter fields produce some important changes relatively to ref. $[14,15,16]$. In particular, the structure of the equations is different as expressed in (3.27)-(3.28) and namely in (3.31).

Let us also point that for frozen scalar fields, i.e., such that $e^{K / 2} P(\phi) \equiv$ const we would obtain a scenario similar to an effective cosmological constant term. In fact, the equations will turn to be familiar to the ones in $[14,15,16]$. Hence, from the asumption $\phi, \bar{\phi}=$ const we would be led again to $\Psi=0$.

Nevertheless, we should stress that the main (and perhaps, solely) conclusions from the case $\mathcal{A}$ is that the adequacy of Ansatz (3.11) has severe limitations. Again, it seems paradoxical that for all degrees of freedom of the Bianchi-IX (diagonal) model with supermatter and analytical potential, the constraints imply that $\Psi=0$ for some simplified assumptions. The proper answer to this situation would have to be addressed from the point of view ref. [23,24]. The Ansatz for $\Psi$ is definitevely incomplete as far as chirality breaking/mode mixing (non-conservation of fermion number in the sense of $[23,24]$ ) is concerned. The results in this subsection can be be taken as another example strengthening 
the arguments of R. Graham and A. Csordás [23,24], although expression (3.11) may still be useful to indicate new possible solutions.

Case $\mathcal{B} \cdot P(\phi)=0$

Let us now consider the case when we choose the analytical potential to be identically zero. The analysis of our Bianchi-IX model with supermatter will turn out to be simpler then in the previous subsection. Moreover, we will follow closely some arguments and framework presented in $[9-12,18,21,25]$.

As we can see either from the equations directly obtained from $S_{A} \Psi=0, \bar{S}_{A^{\prime}} \Psi=$ 0 or from their representation in the tables 1 and 2 , we have self-contained groups of equations relating the 15 wave function coefficients (in the case of $P(\Phi) \neq 0$ that is not true). This applies to 3 groups involving $\left(A, B_{1}, B_{2}, C_{1}, Z_{1}\right),\left(G_{1}, D_{1}, D_{2}, E_{1}, Z_{2}\right)$ and $\left(H_{1}, F_{1}, F_{2}, I_{1}, Z_{3}\right)$. Moreover, notice in particular that the equation corresponding to the terms linear in $\beta, \gamma, \bar{\chi}$ in $\bar{S}_{A^{\prime}} \psi=0$ and $\beta \overline{\chi \chi}(\gamma \gamma)^{2}, \beta \beta \bar{\chi}(\gamma \gamma)^{2}$ in $S_{A} \Psi=0$ completly determine the coefficients $A$ and $I_{1}$. Moreover, $A$ and $I_{1}$ do not appear in any other equation. We have then

$$
\begin{gathered}
A=f(\phi) e^{-\frac{8 \pi^{2}}{\hbar}\left[a_{1}^{2}+a_{2}^{2}+a_{3}^{2}\right]}, \\
I_{1}=k(\bar{\phi}) e^{\frac{8 \pi^{2}}{\hbar}\left[a_{1}^{2}+a_{2}^{2}+a_{3}^{2}\right]} e^{-2 \pi^{2} \phi \bar{\phi}} .
\end{gathered}
$$

The coefficients $G_{1}, H_{1}$ share a similar property with respect to the $\beta \beta \bar{\chi}, \beta \overline{\chi \chi}$ equations from $S_{A} \Psi=0$ and $\beta \beta \bar{\chi} \gamma \gamma, \beta \overline{\chi \chi} \gamma \gamma$ from $S_{A} \Psi=0$, respectively. However, the $\beta \bar{\chi} \gamma$ and $\beta \bar{\chi} \gamma(\gamma \gamma)$ terms in $S_{A} \Psi=0$ impose that $G_{1}$ also appear together with $Z_{2}, D_{2}$ and that $H_{1}$ is together with $Z_{3}, F_{2}$ in independent expressions. This particular property will allow to determine that $G_{1}$ and $I_{1}$ from $Z_{2}, D_{1}$ and $Z_{3}, F_{2}$, respectively.

The equations involving $B_{1}, B_{2}, C_{1}, Z_{1}$ can also be said to be self-contained in the same sense that they involve only these coeficients and no other. Moreover, these coefficients do not occur in any other equations. This can be easily checked, namely from the equations for the terms linear in $\beta, \bar{\chi}$ in $S_{A} \Psi=0, \beta \beta \bar{\chi}$ and $\beta \overline{\chi \chi}$ in $\bar{S}_{A^{\prime}} \Psi=0, \beta \beta \gamma$ in $\bar{S}_{A^{\prime}} \Psi=0$, $\bar{\chi} \gamma \gamma, \beta \gamma \gamma, \gamma \gamma \gamma$ in $\bar{S}_{A^{\prime}} \Psi=0, \gamma$ in $S_{A} \Psi=0$. The previous ones in $\bar{\chi} \gamma \gamma, \beta \gamma \gamma, \gamma \gamma \gamma, \gamma$ just 
involve $C_{1}$. All the others just have $B_{1}, B_{2}, Z_{1}$. However, the $\beta \bar{\chi} \gamma$ equation in $\bar{S}_{A^{\prime}} \Psi=0$ mixes $B_{1}, C_{1}, Z_{1}$. Actually, is the only equation which mixes $C_{1}$ with the remaining bosonic coefficients in the corresponding group.

The same structure of equations and relations between coefficients can be easily checked to occur as well to the subsets involving $D_{1}, D_{2}, E_{1}, Z_{2}$ and $F_{1}, F_{2}, I_{1}, Z_{3}$. Notice again that for the first group, the equations from $\beta(\gamma \gamma)^{2}, \bar{\chi}(\gamma \gamma)^{2}$ in $S_{A} \Psi=0$ and $\gamma \gamma \gamma$ in $S_{A} \Psi=0$ involve only $E_{1}$ and are enough to determine it. Moreover, the $\beta \bar{\chi} \gamma(\gamma \gamma)$ term in $\bar{S}_{A^{\prime}} \Psi=0$ relates $Z_{2}, D_{1}, E_{1}$.

We now proceed to analyse the groups of equations which includes $B_{1}, B_{2}, C_{1}, Z_{1}$. We also include the redefinitions $B_{1} \rightarrow i B, B_{2} \rightarrow B_{2}$ to simplify the results (cf. ref. [10$12,18,25])$. The analysis of the remaining groups is similar, as stated above. The $\beta \beta \bar{\chi}$, $\beta \overline{\chi \chi}$ equations from $\bar{S}_{A^{\prime}} \Psi=0$ and $\beta, \bar{\chi}$ equations from $S_{A} \Psi=0$ give, respectively,

$$
\begin{aligned}
& 4 \hbar^{2} \frac{\partial B_{1}}{\partial \bar{\phi}}+8 \pi^{2} \phi B_{1}+\frac{3 \hbar^{2}}{4} a_{i} \frac{\partial Z_{1}}{\partial a_{i}}-\frac{3 \hbar^{2}}{4 \sqrt{2}} Z_{1}+4 \pi^{2} \hbar a_{1} a_{2} a_{3}\left(\frac{a_{1}}{a_{2} a_{3}}+\frac{a_{2}}{a_{1} a_{3}}+\frac{a_{3}}{a_{1} a_{2}}\right) Z_{1}=0 \\
& \frac{\hbar^{2}}{4} a_{i} \frac{\partial B_{2}}{\partial a_{i}}+4 \pi^{2} \hbar a_{1} a_{2} a_{3}\left(\frac{a_{1}}{a_{2} a_{3}}+\frac{a_{2}}{a_{1} a_{3}}+\frac{a_{3}}{a_{1} a_{2}}\right) B_{2}-\frac{1}{4} B_{2}-2 \hbar^{2} \frac{\partial Z_{1}}{\partial \bar{\phi}}-4 \pi^{2} \hbar^{2} \phi Z_{1}=0 \\
& \frac{\hbar^{2}}{4} a_{i} \frac{\partial B_{1}}{\partial a_{i}}-4 \pi^{2} \hbar a_{1} a_{2} a_{3}\left(\frac{a_{1}}{a_{2} a_{3}}+\frac{a_{2}}{a_{1} a_{3}}+\frac{a_{3}}{a_{1} a_{2}}\right) B_{1}+2 \hbar^{2} \frac{\partial Z_{1}}{\partial \phi}+4 \pi^{2} \bar{\phi} Z_{1}=0 \\
& 4 \hbar^{2} \frac{\partial B_{2}}{\partial \phi}+8 \pi^{2} \bar{\phi} B_{2}-\frac{3 \hbar^{2}}{4} a_{i} \frac{\partial Z_{1}}{\partial a_{i}}+\frac{3 \hbar^{2}}{4 \sqrt{2}} Z_{1}+4 \pi^{2} \hbar a_{1} a_{2} a_{3}\left(\frac{a_{1}}{a_{2} a_{3}}+\frac{a_{2}}{a_{1} a_{3}}+\frac{a_{3}}{a_{1} a_{2}}\right) Z_{1}=0
\end{aligned}
$$

We have used $a_{i}, i=1,2,3$, to denote any operation with respect to $\left(a_{1}, a_{2}, a_{3}\right)$. Making the substitution $B_{1}=\tilde{B}_{1} \exp \left(-2 \pi^{2} \phi \bar{\phi}\right), Z_{1}=\tilde{Z}_{1} \exp \left(-2 \pi^{2} \phi \bar{\phi}\right)$ and $B_{2}=\tilde{B}_{2} \exp \left(-2 \pi^{2} \phi \bar{\phi}\right)$ $[25,40]$, the above four equations become

$$
\begin{aligned}
& 4 \frac{\partial \tilde{B}_{1}}{\partial \bar{\phi}}+\frac{3 a_{i}}{4} \frac{\partial \tilde{Z}_{1}}{\partial a_{i}}-\frac{3}{4 \sqrt{2}} \tilde{Z}_{1}+4 \pi^{2} \hbar^{-1} a_{1} a_{2} a_{3}\left(\frac{a_{1}}{a_{2} a_{3}}+\frac{a_{2}}{a_{1} a_{3}}+\frac{a_{3}}{a_{1} a_{2}}\right) \tilde{Z}_{1}=0, \\
& 4 \frac{\partial \tilde{B}_{2}}{\partial \phi}-\frac{3 a_{i}}{4} \frac{\partial \tilde{Z}_{1}}{\partial a_{i}}+\frac{3}{4 \sqrt{2}} \tilde{Z}_{1}+4 \pi^{2} \hbar^{-1} a_{1} a_{2} a_{3}\left(\frac{a_{1}}{a_{2} a_{3}}+\frac{a_{2}}{a_{1} a_{3}}+\frac{a_{3}}{a_{1} a_{2}}\right) \tilde{Z}_{1}=0,
\end{aligned}
$$




$$
\begin{gathered}
2 \frac{\partial \tilde{Z}_{1}}{\partial \bar{\phi}}-\frac{3}{4} a_{i} \frac{\partial \tilde{B}_{2}}{\partial a_{i}}-4 \pi^{2} \hbar^{-1} a_{1} a_{2} a_{3}\left(\frac{a_{1}}{a_{2} a_{3}}+\frac{a_{2}}{a_{1} a_{3}}+\frac{a_{3}}{a_{1} a_{2}}\right) \tilde{B}_{2}+\frac{1}{4} a_{1} a_{2} a_{3} \tilde{B}_{2}=0 \\
2 \frac{\partial \tilde{Z}_{1}}{\partial \phi}+\frac{1}{4} a_{i} \frac{\partial \tilde{B}_{1}}{\partial a_{i}}-2 \pi^{2} \hbar^{-1} a_{1} a_{2} a_{3}\left(\frac{a_{1}}{a_{2} a_{3}}+\frac{a_{2}}{a_{1} a_{3}}+\frac{a_{3}}{a_{1} a_{2}}\right) \tilde{B}_{1}=0 .
\end{gathered}
$$

We will now use $(3.35 \mathrm{a}),(3.35 \mathrm{~d})$ and $(3.35 \mathrm{~b}),(3.35 \mathrm{c})$ to eliminate $\tilde{B}_{1}$ or $\tilde{B}_{2}$ to get a differential equation for $\tilde{Z}_{1}$. Let us take $(3.35 \mathrm{~b}),(3.35 \mathrm{c})$. Applying $\frac{\partial}{\partial \phi}$ to $(3.35 \mathrm{c})$, using (3.35b) for $\frac{\partial B_{2}}{\partial \phi}$, we obtain

$$
\begin{aligned}
& 2 \frac{\partial \tilde{Z}_{1}}{\partial \bar{\phi} \partial \phi}-\frac{3 a_{i}}{4^{3}} \frac{\partial}{\partial a_{i}}\left(a_{j} \frac{\partial \tilde{Z}_{1}}{\partial a_{j}}\right)+\frac{9}{4^{3} \sqrt{2}} a_{i} \frac{\partial \tilde{Z}_{1}}{\partial a_{i}} \\
& +\left[4 \pi^{4} \hbar^{-2}\left(a_{1} a_{2} a_{3}\left(\frac{a_{1}}{a_{2} a_{3}}+\frac{a_{2}}{a_{1} a_{3}}+\frac{a_{3}}{a_{1} a_{2}}\right)\right)^{2}\right. \\
& \left.-\pi^{2} \hbar^{-1} \frac{3+\sqrt{2}}{4 \sqrt{2}} a_{1} a_{2} a_{3}\left(\frac{a_{1}}{a_{2} a_{3}}+\frac{a_{2}}{a_{1} a_{3}}+\frac{a_{3}}{a_{1} a_{2}}\right)-\frac{3}{4^{3} \sqrt{2}}\right] \tilde{Z}_{1}=0
\end{aligned}
$$

We proceed similarly for the equations (3.35a), (3.35d) but we notice that relatively to ref. $[10,12,25]$, the term in $(3.35 \mathrm{~d})$ linear in $\tilde{B}_{1}$ is absent. This is due to the ordering we choose from the full theory constraints. Had we chosen a different ordering procedure for the $S_{A}$ quantum constraint, as the one in ref. [10,12,21] or even as in [11] (see also ref. [25]), the last column in table 1 for the $\beta$ row will have a bosonic coefficient $B_{1}$. However, it is easy to see from the above equations that the coefficients of the third, fifth and sixth terms in (3.36) will differ from the ones in the equation for $Z_{1}$ which we derive from the remaining equations $(3.35 \mathrm{a}),(3.35 \mathrm{~d})$. Consistency than implies that $\tilde{Z}_{1}=0$. Consequently, the equations for terms with combinations of only $\beta$ and $\gamma$ involve just $B_{1}$ and $C_{1}$. These are then as the ones in the case of a Bianchi-IX with $\Lambda=0$ and no supermatter [7]. The only possible solution of these equations with respest to $a_{1}, a_{2}, a_{3}$ is the trivial one, i.e., $B_{1}=C_{1}=0$. The equations corresponding to $\bar{\chi}$ and combinations of it with $\beta$ or $\gamma$ would give, with $B_{1}=C_{1}=Z_{1}=0$, the dependence of $B_{2}$ on $a_{1}, a_{2}, a_{3}, \phi$. This correspond to

$$
B_{2}=h(\bar{\phi}) a_{1} a_{2} a_{3} e^{-\frac{8 \pi^{2}}{\hbar}\left[a_{1}^{2}+a_{2}^{2}+a_{3}^{2}\right]} e^{-2 \pi^{2} \phi \bar{\phi}} .
$$

This pattern repeats itself in a similar way when we consider the two groups involving $D_{1}, D_{2}, E_{1}, Z_{2}$ and $F_{1}, F_{2}, Z_{3}$. In particular, notice that from the $\beta \bar{\chi} \gamma \gamma \gamma$ and $\beta \bar{\chi} \gamma$ terms in $S_{A} \Psi=0$ we get $E_{1}=G_{1}=H_{1}=0$ from $Z_{2}=D_{2}=0, Z_{2}=D_{1}=0$, and $Z_{3}=F_{1}=0$. 
Hence, besides $A$ and $I_{1}$, only $B_{2}$ and $F_{2}$ will be different from zero. We can write than for the solution of the constraints, using the Ansatz (3.13),

$$
\begin{aligned}
\Psi & =f(\phi) e^{\frac{8 \pi^{2}}{\hbar}\left[a_{1}^{2}+a_{2}^{2}+a_{3}^{2}\right]} \\
& +h(\bar{\phi}) a_{1} a_{2} a_{3} e^{\left[-a_{1}^{2}-a_{2}^{2}-a_{3}^{2}\right]} e^{-2 \pi^{2} \phi \bar{\phi}} \bar{\chi}_{A} \bar{\chi}^{A} \\
& +g(\phi) a_{1} a_{2} a_{3} e^{\frac{8 \pi^{2}}{\hbar}\left[a_{1}^{2}+a_{2}^{2}+a_{3}^{2}\right]} e^{-2 \pi^{2} \phi \bar{\phi}} \beta_{A} \beta^{A}\left(\gamma_{B C D} \gamma^{B C D}\right)^{2} \\
& +k(\bar{\phi}) e^{\frac{8 \pi^{2}}{\hbar}\left[a_{1}^{2}+a_{2}^{2}+a_{3}^{2}\right]} \bar{\chi}_{A} \bar{\chi}^{A} \beta_{E} \beta^{E}\left(\gamma_{B C D} \gamma^{B C D}\right)^{2}
\end{aligned}
$$

\section{Conclusions, Discussions and Outlook}

In this paper we have studied the quantization of a Bianchi type IX (diagonal) model in $\mathrm{N}=1$ supergravity in the presence of supermatter. The supermatter content was constituted by a scalar multiplet, i.e., a pair of complex scalar fields together with their odd (anti-

commuting) spin- $\frac{1}{2}$ fields partners. The corresponding Kähler geometry was chosen to be a two-dimensional flat one.

Our approach can be characterized as twofold. On the one hand, we applied directly the quantum constraints of the full theory of $\mathrm{N}=1$ supergravity with supermatter [54] subject to a (diagonal) Bianchi type-IX Ansätze for the fields. On the other hand, we restricted ourselves to a simple Lorentz invariant Ansatz for the wave function of the universe, $\Psi$. In particular, only the irreducible spin $\frac{1}{2}, \frac{3}{2}$ mode components of the fermionic fields have been considered. We then analysed two possible cases, namely when the scalar field dependent analytical potential in the supermatter content was either arbitrary or identically set to zero.

In the former, our main (and perhaps, solely) conclusion was that the adequacy of our Ansatz for $\Psi$ is severly limited as far as chirality breaking, mode mixing or non conservation of fermion number (in the sense of [23,24]) are concerned. Following [23,24], it seems paradoxical that for all the degrees of freedom present in the Bianchi type-IX with supermatter the constraints imply $\Psi=0$ for some simplifying assumptions. These include either putting the analytical potential term constant and different from zero (as an effective cosmological constant) or choosing some particular simple solutions for specific bosonic amplitude coefficients in the Ansatz of $\Psi$. In a more general setting, no easy 
way is apparent of obtaining an analytical solution for the full set of equations. In fact, exponential terms as $e^{\phi \bar{\phi}}$ lead to serious difficulties. However, the possibility to have nontrivial states for $\Psi$ could not be ruled out at cubic order in $\psi_{i}^{A}$ as in ref. $[14,15,16]$. In fact, the presence of the matter degrees of freedom together with the anisotropic gravitational ones and the spin- $\frac{3}{2}$ modes of the gravitino allows one to speculate on that possibility.

For $P(\phi)$, however, we found out that $\Psi$ had indeed a very simple form. Namely, the only non zero components of the wave function can be found in the sectors with no fermions (bosonic) and in three other sectors, more precisely filled with just the spin- $\frac{1}{2}$ fermionic partners of the scalar field, another filled with just the spin- $\frac{1}{2}$ and $\frac{3}{2}$ mode components of the spatially homogeneous gravitino field and finally one totally filled with spin- $\frac{1}{2}$ fermionic partners of the scalar field as well as the the spin- $\frac{1}{2}$ and $\frac{3}{2}$ mode components of the gravitino field. More precisely, we obtained

$$
\begin{aligned}
\Psi & =f(\phi) e^{-\frac{8 \pi^{2}}{\hbar}\left[a_{1}^{2}+a_{2}^{2}+a_{3}^{2}\right]} \\
& +h(\bar{\phi}) a_{1} a_{2} a_{3} e^{-\frac{8 \pi^{2}}{\hbar}\left[a_{1}^{2}+a_{2}^{2}+a_{3}^{2}\right]} e^{-2 \pi^{2} \phi \bar{\phi}} \bar{\chi}_{A} \bar{\chi}^{A} \\
& +g(\bar{\phi}) a_{1} a_{2} a_{3} e^{\frac{8 \pi^{2}}{\hbar}\left[a_{1}^{2}+a_{2}^{2}+a_{3}^{2}\right]} e^{-2 \pi^{2} \phi \bar{\phi}} \beta_{A} \beta^{A}\left(\gamma_{B C D} \gamma^{B C D}\right)^{2} \\
& +k(\bar{\phi}) e^{\frac{8 \pi^{2}}{\hbar}\left[a_{1}^{2}+a_{2}^{2}+a_{3}^{2}\right]} \bar{\chi}_{A} \bar{\chi}^{A} \beta_{E} \beta^{E}\left(\gamma_{B C D} \gamma^{B C D}\right)^{2} .
\end{aligned}
$$

The simple semi-classical form of the bosonic amplitude coeficients in $\Psi$ above suggests that we might find among them the Hartle-Hawking (no-boundary) state [45] or the (ground) wormhole quantum state of the theory [46], both of the form $P e^{-I}$ where $I$ is a certain Euclidian action. The wormhole state should correspond to an asymptotic 4Euclidian classical solution, which is outwards to a 3-geometry, required to be regular at small 3-geometries (the interior boundary) and to die away rapidly at large 3-geometries [46]. For the Hartle-Hawking state, we require a regular solution of the classical field equations with metric (3.3) defined on the outer boundary [45].

The arbitrary functions $f, g, h, k$ of $\phi, \bar{\phi}$ do not allow to conclude unambiguously that in any of the fermionic sectors the corresponding bosonic amplitudes will die away for large 3-geometries and $\phi, \bar{\phi}$ at infinity. Moreover, a wormhole state for a FRW case would have the form prefactor $\times e^{-3 a^{2}+3 a^{2} \cosh (\rho)}$ where $\phi=\rho e^{i \theta}$ and one would expect a simple generalization of it to the Bianchi-IX case. Hence it seems that we cannot find a wormhole 
ground state. However, a similar issue for the case of a FRW model was addressed recently (see ref. [62] for details) and where a wormhole basis may still be constructed.

With regard to the Hartle-Hawking state, the same arguments in [7] can be used to show that we cannot identify such type of solution in (3.37). According to Graham and Luckock [8], another definition of homogeneity conditions for the gravitino field could lead us to obtain instead

$$
\begin{aligned}
\Psi & =f(\phi) e^{-\frac{8 \pi^{2}}{\hbar}\left[a_{1}^{2}+a_{2}^{2}+a_{3}^{2}+2\left(a_{2} a_{3}+a_{1} a_{3}+a_{1} a_{2}\right)\right]} \\
& +h(\bar{\phi}) a_{1} a_{2} a_{3} e^{-\frac{8 \pi^{2}}{\hbar}\left[a_{1}^{2}+a_{2}^{2}+a_{3}^{2}+2\left(a_{2} a_{3}+a_{1} a_{3}+a_{1} a_{2}\right)\right]} e^{-2 \pi^{2} \phi \bar{\phi}} \bar{\chi}_{A} \bar{\chi}^{A} \\
& +g(\phi) a_{1} a_{2} a_{3} e^{\frac{8 \pi^{2}}{\hbar}\left[a_{1}^{2}+a_{2}^{2}+a_{3}^{2}-2\left(a_{2} a_{3}+a_{1} a_{3}+a_{1} a_{2}\right)\right]} e^{-2 \pi^{2} \phi \bar{\phi}} \beta_{A} \beta^{A}\left(\gamma_{B C D} \gamma^{B C D}\right)^{2} \\
& +k(\bar{\phi}) e^{\frac{8 \pi^{2}}{\hbar}\left[a_{1}^{2}+a_{2}^{2}+a_{3}^{2}-2\left(a_{2} a_{3}+a_{1} a_{3}+a_{1} a_{2}\right)\right]} \bar{\chi}_{A} \bar{\chi}^{A} \beta_{E} \beta^{E}\left(\gamma_{B C D} \gamma^{B C D}\right)^{2},
\end{aligned}
$$

where we could identify the Hartle-Hawking state.

With respect to the models considered so far within the more general $\mathrm{N}=1$ supergravity theory with supermatter $[18,21,25,26,54]$, we would expect our locally supersymmetric (diagonal) Bianchi-IX model coupled to a scalar supermultiplet to bear instead important differences. In fact, the models in $[18,21,25,26]$ were FRW ones and consequently the gravitino fields were required to to be (severly) restricted to their spin- $\frac{1}{2}$ modes. However, the presence now of anisotropic gravitational degrees of freedom and hence of the spin- $\frac{3}{2}$ modes of the gravitinos could play an important role. Moreover, it would bring our minisuperspace model closer to the features of a full theory of $\mathrm{N}=1$ supergravity with supermatter in spite of the drastic inhomogeneous modes truncation. Our results corresponded to "straightforward" anisotropic generalization of the previous works in the same line of research $[18,21,25,26]$. Nevertheless, we could not avoid some problems. In particular, the absence of the Hartle-Hawking and wormhole states. In the following, we would like to discuss (and somehow, to speculate) what may be the possible reasons for that.

Firstly, we have obtained our differential equations by applying the quantum constraints of the full theory of $\mathrm{N}=1$ supergravity with supermatter subject a the Bianchi-IX (diagonal) Ansatz. This has been used previously $[6,7,14,15,16]$ and in agreement with other approaches $[34,35,36]$. However, there are some points which could be of some importance. We begin by noticing that in this paper as well as in others following the same approach $[1,6,7,14,15,16]$, contorsion terms are absent in the full theory supersymmetry 
constraints. This can be related to the inclusion of the Lorentz constraints in the spatially integrated Hamiltonian through the Lagrange multipliers $\omega_{A B}^{0}, \bar{\omega}_{A^{\prime} B^{\prime}}^{0}$. However, in dimensional-reduced cases with supermatter $[10,11,12,18,21,25]$ contorsion cubic fermionic terms in the gravitino field are present. In ref. $[9,10]$ when no supermatter was considered, a redefinition of Lagrange multipliers was introduced in order to obtain a simple form for the generators in the Hamiltonian and their Dirac brackets; subsequetly the contorsion cubic terms dissapeared. It may be that when supermatter is present such redefinitions cannot be straigthforwardly extended from the pure $\mathrm{N}=1$ supergravity case, either in the full theory or from a dimensional reduction approach (see [43] and sections 5,6 in [10]).

Secondly, we have used an overly restrictive Ansatz for $\Psi$ in the sense that the Lorentz invariant sectors have been constructed without the irreducible spin components of the gravitational degrees of freedom $[23,24]$. We are conscient of this fact and our purpose was to obtain an indication of what will be the picture in the "new" framework as explained by R. Graham and A. Csordás [23,24]. Our arguments were as follows. Both the bosonic and fermionic filled sectors amplitudes in [23,24] could be obtained from Lorentz invariant sectors containing only the irreducible spin components of the gravitino field. Moreover, the solutions for the middle sectors (absent in the old framework) were allready present in [8], using the old Ansatz for $\Psi$ together with a different homogeneity condition for gravitino. The old framework uses a set of coupled first order differential equations while the solutions for middle states in [23,24] (where the Hartle-Hawking state was properly identified) depend on our ability to solve a (second-order) Wheeler-DeWitt type of equation. Hence, we hoped that the old approach could still be of some utility as far as locally supersymmetric models with supermatter are concerned.

It seems though from our analysis and discussion hereby presented, that when supermatter is present one cannot simply expect to follow a somewhat simple relation between the old and new frameworks. Perhaps the approach in [23,24] properly applied to our Bianchi-IX model could be able to find out the Hartle-Hawking states in other middle sectors. However, the absence of a wormhole state is another issue to be addressed [62]. Either a fundamental piece in constructing the reduced theory (following any of the possible approaches) has been neglected or then we would have to conclude that when the more general theory of $\mathrm{N}=1$ supergravity with supermatter [54] is rightfully considered the 
wormhole picture may not be the ground state.

\section{ACKNOWLEDGEMENTS}

The author is thankful to A.D.Y. Cheng for many pleasant discussions and for sharing his points of view and to S.W. Hawking for helpful suggestions and conversations. Discussions with O. Obregon and R. Graham are also acnowledged. The author gratefully acknowledges the Instituto de Fisica de la Universidad de Guanajuato, Mexico, for supporting part of his 3 weeks visit as well as the support of a Human Capital and Mobility (HCM) Fellowship from the European Union (Contract ERBCHBICT930781).

\section{REFERENCES}

[1] P.D. D'Eath, Phys. Rev. D 29, 2199 (1984).

[2] A. Macias, O. Obrégon and M. Ryan, Class. Quantum Grav. 4, 1477 (1987).

[3] O. Obrégon, J. Socorro and J. Benitez, Phys. Rev. D 47, 4471 (1993).

[4] J. Socorro, O. Obrégon and A. Macias, Phys. Rev. D 45, 2026 (1992).

[5] A. Macias, O. Obrégon and J. Socorro, Int. J. Mod. Phys. A8, 4291 (1993).

[6] P.D. D'Eath, S.W. Hawking and O. Obregón, Phys. Lett. 300B, 44 (1993).

[7] P.D. D'Eath, Phys. Rev. D 48, 713 (1993).

[8] R. Graham and H. Luckock, Phys. Rev. D 49, R4981 (1994).

[9] P.D. D'Eath and D.I. Hughes, Phys. Lett. 214B, 498 (1988).

[10] P.D. D'Eath and D.I. Hughes, Nucl. Phys. B 378, 381 (1992).

[11] L.J. Alty, P.D. D'Eath and H.F. Dowker, Phys. Rev. D 46, 4402 (1992).

[12] D.I. Hughes, Ph.D. thesis, University of Cambridge (1990), unpublished.

[13] M. Asano, M. Tanimoto and N. Yoshino, Phys. Lett. 314B, 303 (1993).

[14] P.D. D'Eath, Phys. Lett. B320, 20 (1994).

[15] A.D.Y. Cheng, P.D. D'Eath and P.R.L.V. Moniz, Phys. Rev. D49 (1994) 5246.

[16] A.D.Y. Cheng, P.D. D'Eath and P.R.L.V. Moniz, Gravitation and Cosmology 1 (1995) 1

[17] A.D.Y. Cheng, P.D. D'Eath and P.R.L.V. Moniz, DAMTP-Report February R94/13, submitted to Physical Review D.

[18] A.D.Y. Cheng, P.D. D'Eath and P.R.L.V. Moniz, Gravitation and Cosmology - Proceedings 1 (1995) 12 
[19] S. Carroll, D. Freedman, M. Ortiz and D. Page, Nuc. Phys. B423, 3405 (1994).

[20] S. Carroll, D. Freedman, M. Ortiz and D. Page, Bosonic physical states in N=1 supergravity? in: Procedings of the MG7 Meeting in General Relativity, Stanford, July 1994 (World Scientific), gr-qc 9410005.

[21] A.D.Y. Cheng, P.D. D'Eath and P.R.L.V. Moniz, Class. Quantum Grav., to appear; Quantization of a FRW model in $N=1$ supergravity with gauged supermatter in: Proceedings of the First Mexican School in Gravitation and Mathematical Physics, Guanajuato, December 12-16, 1994 (9503009).

[22] H. Luckock and C. Oliwa, Sidney University Report, (gr-qc 9412028), accepted for publication in Phys. Rev. D.; C. Oliwa, M.Sc. thesis, University of Sidney (1994), unpublished.

[23] R. Graham and A. Csordás, Nontrivial fermion states in supersymmetric minisuperspace, in: Proceedings of the First Mexican School in Gravitation and Mathematical Physics, Guanajuato, Mexico, December 12-16, 1994 (9503054 ).

[24] R. Graham and A. Csordás, Phys. Rev. Lett. 74 (1995) 4926.

[25] \& Int. J. Mod. Phys. D4, No.2 April (1995) - to appear.

[26] A.D.Y. Cheng, unpublished report.

[27] R. Graham, Phys. Rev. Lett. 67, 1381 (1991).

[28] R. Graham and J. Bene, Phys. Lett. B 302, 183 (1993).

[29] R. Graham, Phys. Rev. D 48, 1602 (1993).

[30] R. Graham and J. Bene, Phys. Rev. D49, 799 (1994).

[31] R. Graham and H. Luckock, Phys. Rev. D 49, 2786 (1994).

[32] T. Jacobson, Class. Quantum Grav. 5, 923, (1988).

[33] T. Sano and J. Shiraishi, Nucl. Phys. B410, 423, (1993).

[34] O. Obrégon, J. Pullin and M. Ryan, Phys. Rev. D 48, 5642 (1993).

[35] R. Capovilla and J. Guven, Class. Quantum Grav. 11, 1961 (1994).

[36] R. Capovilla and O. Obregon, Phys. Rev. D49 (1994) 6562.

[37] H.-J. Matschull, Class. Quantum Grav. 11 (1994) 2395.

[38] H.-J. Matschull and H. Nicolai, Jour. Geom. Phys. 11, 15 (1993); Nucl. Phys. B 411, 609 (1994); B. de Wit, H.-J. Matschull and H. Nicolai, Phys. Lett. B 318, 115 (1993). 
[39] T. Sano, hep-th 9211103; T. Sano and J. Shiraishi, Nuc. Phys. B410 (193) 423.

[40] H. Kunimoto and T. Sano, Int. J. Mod. Phys. D1 (1993) 559.

[41] P. V. Moniz, Supersymmetric Quantum Cosmology, work in preparation.

[42] C. Teitelboim, Phys. Rev. Lett. 38, 1106 (1977).

[43] M. Pilati, Nuc. Phys. B 132, 138 (1978).

[44] M. Ryan Jr. and L. Shepley, "Homogeneous Relativistic Cosmologies" (PUP, Princeton 1972).

[46] S.W. Hawking and D.N. Page, Phys. Rev. D 42, 2655 (1990).

[45] J.B. Hartle and S.W. Hawking, Phys. Rev. D 28, 2960 (1983).

[47] C. Teitelboim, Phys. Rev. D 25, 3159 (1982)

[48] A.D.Y. Cheng, private communication.

[49] R. Graham, private communication.

[50] P. van Nieuwenhuizen, Phys. Rep. 68 (1981) 189; D. Freedman and A. Das, Nuc. Phys. B 120, 221 (1977).

[51] S. Ferrara and P. van Nieuwenhuizen, Phys. Rev. Lett. 37, 1669 (1976).

[52] D. Freedman, Phys. Rev. Lett. 38, 105 (1977).

[53] \&, work in progress.

[54] J. Wess and J. Bagger, Supersymmetry and Supergravity, 2nd. ed. (Princeton University Press, 1992).

[55] A. Das. M. Fishler and M. Rocek, Phys. Lett. B 69, 186 (1977).

[56] O. Obrégon, IFUG preprint-1995.

[57] O. Obrégon, private communication.

[58] J.E. Nelson and C. Teitelboim, Ann. Phys. (N.Y.) 116 (1978) 86.

[59] P.D. D'Eath and J.J. Halliwell, Phys. Rev. D 35 (1987) 1100.

[60] E. Witten and J. Bagger, Phys. Lett. B 115 (1982).

[61] L.D. Faddeev and A.A. Slavnov, Gauge Fields (Benjamin, Reading, Mass., 1980).

[62] P.V. Moniz, Is there a problem with quantum wormholes in $N=1$ supergravity, essayDAMTP R95/19; The case of the missing wormhole case, talk present in the 6th Moskow Quantum Gravity Seminar.

[63] P.V. Moniz, Locally supersymmetric FRW model with Yang-Mills fields, in preparation. 